\title{
A potential role for the gut microbiota in the specialisation of Drosophila sechellia to its toxic host noni (Morinda citrifolia)
}

\author{
Heys $C^{1,2}$, Fisher $A M^{1}$, Dewhurst $A D^{1}$, Lewis $Z^{1 *}$ and Lizé $A^{1,3}$
}

${ }^{1}$ Institute of Integrative Biology/School of Life Sciences, University of Liverpool, Liverpool, L69 7ZB, UK

${ }^{2}$ Institute of Biodiversity, Animal Health, and Comparative Medicine, University of Glasgow, Glasgow, G12 8QQ, UK

3UMR CNRS 6553 ECOBIO, University of Rennes 1, 35042, Rennes, FRANCE

*Author for correspondence: Z.Lewis@liverpool.ac.uk

\section{Abstract}

Adaptation to a novel food source can have significant evolutionary advantages. The fruit fly, Drosophila sechellia, is a specialist of the toxic plant noni (Morinda citrifolia). Little is known as to how $D$. sechellia has become resistant to the toxins in the fruit comprised predominantly of octanoic acid - but to date, the behavioural preferences for the fruit and genetic architecture underlying them, have been well studied. Here, we examine whether the gut microbiota could have played a role in adaptation to the fruit. In the first series of experiments, we examine the gut microbiota of wild-type, laboratory reared flies and characterise the gut microbiota when reared on the natural host plant, versus a standard Drosophila diet. We show a rapid transition in the core bacterial diversity and abundance within this species and discover sole precedence of Lactobacillus plantarum when reared on M. citrifolia. We also discover that flies reared on a laboratory diet are more likely to carry bacterial pathogens such as Bacillus cereus, although their function in Drosophila is unknown. Flies reared on a laboratory diet have a significantly reduced weight but with no impact on the risk of death before adulthood, when compared to the wild noni diet. In the second series of experiments, 
we examine the potential role of the gut microbiota in adaptation to octanoic acid resistance in this species and its sister species, Drosophila melanogaster, to which the fruit is usually fatal. We use a combination of methods to analyse resistance to octanoic acid by conducting life history analysis, behavioural assays and bacterial analysis in both $D$. sechellia and $D$. melanogaster. We find that by creating experimental evolution lines of $D$. melanogaster supplemented with gut microbiota from $D$. sechellia, we can decrease $D$. melanogaster aversion to octanoic acid, with the flies even preferring to feed on food supplemented with the acid. We suggest this represents the first step in the evolutionary and ecological specialisation of $D$. sechellia to its toxic host plant, and that the gut microbiota, Lactobacillus plantarum in particular, may have played a key role in host specialisation. 


\section{Introduction}

Many studies have examined the complex relationships between animals and plants (reviewed in Herrera and Pellmyr, 2009). The dynamic ecological and evolutionary interactions between an animal and its host plant can take many forms. In some cases, insects have even become adapted to living on a toxic plant host, such as Pierinae butterflies that feed on toxic Brassicales (e.g. Edger et al., 2015). Developing resistance to a toxic host plant through specialisation can have a multitude of advantages, including the ability to exploit an otherwise unutilised resource, and there are many ways that insects can overcome the toxins in the host plant. Behavioural and physiological adaptations to exploit toxic resources can drive speciation of insects or other animals (Matsuo et al., 2007). However, it is argued that specialisation to a toxic host does not necessarily result in speciation itself, and therefore the role of ecological specialisation in speciation remains to be proven (Matsuo et al., 2007).

The way in which these specialists adapt to life in a novel environment can occur through a number of different ways, and one is potentially through the gut microbiota (e.g. Bolnick et al., 2014; Morrow et al., 2015). In insects, for example, members of the order Hemiptera have evolved to feed on plant phloem sap - a nutritionally poor diet due to the grossly unbalanced amino acid composition (e.g. Douglas, 1993; Sandström and Moran, 1999; Sandström, 2000). A number of studies have demonstrated that all phloem feeders within this order possess certain symbiotic bacteria that mitigate the effects of this nutritionally poor diet (Buchner 1965; Gündüz and Douglas, 2009). For example, two specialist species of Lepidoptera, Hyles euphorbiae and Brithys crini, feed exclusively on latex-rich Euphorbia sp. and alkaloid-rich Pancratium maritimum, respectively (Vilanova et al., 2016). Metagenomic sequencing has identified that the primary microbiota within the gut is Entereococcus sp., which it is predicted to be responsible for mitigating the effects of these nutrient-poor diets to the host.

The participation of the gut microbiota in the transformation of toxic plant chemicals is an important aspect to be considered when studying insect-plant interactions, and one that is gaining attention (e.g. Genta et al., 2006; Ceja-Navarro et al., 2015). Yet due to the complexity and diversity of gut microorganisms, there is little experimental evidence to support this idea. It was suggested by Douglas (1992) that a possible role of the midgut microbiota is in detoxification of toxic compounds and a study by Genta 
et al., (2006) in Tenebrio molitor highlighted the role of gut microbiota in detoxifying the cell walls of fungi and bacteria that typically inhabit their food source. Similarly, the coffee berry borer (Hypothenemus hampei), the most devastating insect pest of coffee crops worldwide, has adapted to metabolise caffeine - a toxic alkaloid present within coffee plants (Ceja-Navarro et al., 2015). Caffeine is shown to be degraded in the gut via Pseudomonas species, which subsist on caffeine as a sole source of carbon and nitrogen (Ceja-Navarro et al., 2015).

The majority of species within the Drosophila melanogaster species-complex are food generalists and saprophagous, meaning they feed on a variety of decaying plant matter (Rohlfs and Kürschner, 2010). Studies have shown the importance of a diverse diet in creating and maintaining a diverse gut microbiota within $D$. melanogaster, with a diverse gut microbiota increasing survival and reducing development time (Rohlfs and Kürschner, 2010). In comparison, several species within the $D$. melanogaster species-complex have evolved some form of diet specialisation (e.g. Lachaise et al., 1988). One species within this group, Drosophila sechellia, is a specialist of ripe Morinda citrifolia fruit - a toxic fruit commonly known as the Tahitian noni (Jones, 2005). D. sechellia's closely related species, Drosophila simulans, Drosophila mauritiana and $D$. melanogaster are notably repelled by the pungent scent of the fruit and even die upon contact (Legal et al., 1994; Legal et al., 1999). Resistance of $D$. melanogaster to the fruit is shown to be dependent on strain (Legal et al., 1992).

The primary toxins found within noni are octanoic and hexanoic acids (Farine et al., 1996), and numerous studies have focussed on the underlying genetic and molecular mechanisms that have enabled $D$. sechellia to adapt to the fruit (e.g. Harada et al., 2008, 2012; Labeur et al., 2002; Matsuo et al., 2007. However, whether the gut microbiota has played a role in this specialisation has not yet been investigated. Interestingly, Chandler et al. (2011) characterised the microbiota of wild D. sechellia found feeding on the noni and discovered that the gut is dominated by a single Lactobacillales OTU (84\%). This demonstrates the very low bacterial community richness and diversity within this species, particularly when it is compared to its sister species, D. melanogaster, which exhibit greater diversity and carry the bacterial genera Lactobacillus, Acetobacter and Enterococcus (Ryu et al., 2008; Brummel et al., 2004; Ren et al., 2007; Cox and Gilmore, 2007). Further, the composition of an individual's gut microbiota is known to be influenced by pH (see Overend et al., 2016). 
Overend et al. (2016) demonstrated that decreasing the $\mathrm{pH}$ in certain regions of the Drosophila gut can lead to an increased abundance of key members of the gut microbiota - Lactobacillus and Acetobacter. This raises the question whether the very low bacterial richness found in the $D$. sechellia gut when it is feeding on its acidic natural host diet, $M$. citrifolia, is due to the $\mathrm{pH}$ determining the microbiota? Alternatively, potentially the almost exclusive prevalence of this Lactobacillales is caused by the specialism of $D$. sechellia to the host plant, and thus the Lactobacillales acts as a form of detoxifying agent by metabolising the toxic acids found within the noni.

In this study we investigated the role of the gut microbiota on host specialisation in $D$. sechellia. This study is separated into two sections. In the first, we determined the effect that rearing $D$. sechellia on a standard laboratory diet has on the diversity and richness of the gut microbiota. $D$. sechellia are widely kept in the laboratory, but little attention has been paid to the effect that feeding this specialist species a generalist diet has on the resulting gut microbiota. Flies were first reared on a standard Drosophila diet (ASG), then moved onto noni, before being transferred back onto ASG. At each stage, the diversity and abundance of the gut bacteria was measured. We also disentangled the role of $\mathrm{pH}$ on shaping the gut microbiota, from the toxic compounds present in the noni. We introduced a new dietary treatment, salak fruit (Salacca zalacca), with similar nutritional and acidic properties to noni but lacking in the toxic compounds - octanoic and hexanoic acid. We determined the effect that these diets a standard laboratory diet, noni and salak fruit - had on a series of life history traits, including larval, pupal and adult weight and the risk of death before adulthood. We predicted that the gut microbiota would become more simplified on the noni diet, but not the salak or ASG, which would indicate that the gut microbiota plays a role in specialisation to this diet.

In the second part of this experiment, we investigated the effects of differing concentrations of the toxic compound octanoic acid, the main acidic constituent of $D$. sechellia's natural host plant, $M$. citrifolia, on weight, development time, survival, bacterial load and diversity in $D$. sechellia. We also investigated the same effects in $D$. sechellia's sister species, $D$. melanogaster, to determine any differences between a fruit specialist and a fruit generalist species. We analysed both inbred and outbred lines in $D$. melanogaster in order to test the effect of strain on ability to withstand 
octanoic acid exposure. We predicted that $D$. melanogaster would have a reduced weight, development time and survival ability compared to $D$. sechellia, particularly in the inbred strain in which genetic diversity is low. We also predicted that we would observe differences in the diversity and abundance of the gut microbiota of $D$. sechellia compared to $D$. melanogaster, due to $D$. sechellia possessing a more specialised gut microbiota that enable them to withstand high concentrations of octanoic acid. We further examined the role of the gut microbiota in this specialisation by creating experimental evolution lines of $D$. melanogaster supplemented with $D$. sechellia gut microbes. As we hypothesised the gut microbiota may have played a key role in the specialisation in $D$. sechellia, we predicted that after ten generations $D$. melanogaster would be less averse to exposure to octanoic acid.

\section{Materials and methods}

\section{Flies used and general maintenance}

D. sechellia stocks were obtained from the National Drosophila Species Stock Center located in San Diego. Three lines of outbred flies were utilised (lines 0.21, 0.07 and 0.08), that were collected on Cousin Island, Seychelles in 1980 and maintained in the laboratory ever since. All flies were kept and reared at $25^{\circ} \mathrm{C}$ on a $12: 12 \mathrm{hour} \mathrm{light-dark}$ cycle. Flies were kept in standard $75 \times 25 \mathrm{~mm}$ Drosophila vials containing $25 \mathrm{ml}$ of standard Drosophila food composed of yeast/agar/maize/sugar. Flies were moved to new vials every 4 days.

\section{Experiment 1: The changing gut microbiota of $D$. sechellia}

\section{Experimental treatments}

Newly emerged, virgin adult flies were obtained from the stock population and transferred to a new vial containing $25 \mathrm{ml}$ of a standard Drosophila dietary media composed of yeast/sugar/agar/maize (hereon known as ASG). Flies were left to mature on this media for two days before being transferred to a new vial containing the same media ( $\mathrm{N}=30)$. After one week, two males and two females from different vials from each stock line were isolated using carbon dioxide gas anaesthesia. The 
protocol below detailing the bacterial analysis was then followed for these individuals, to determine the gut bacterial load and diversity of flies reared on this diet. These flies formed the "ASG 1" treatment.

The remaining flies were then gently aspirated into fresh vials containing $25 \mathrm{~g}$ of noni and left for one week ( $N=30)$. After this time, two males and two females from different vials from each stock line were again isolated and the same bacterial analysis protocol was followed. This enabled us to determine any changes in the gut microbiota in the same population of flies, that were first reared on a different diet. These flies formed the "Noni" treatment.

Similarly, the remaining flies were again, gently aspirated into fresh vials containing $25 \mathrm{ml}$ of $A S G$ and left for one week $(\mathrm{N}=30)$. After this time, two males and two females from different vials from each stock line were again isolated and the same bacterial analysis protocol was followed. This enabled us to determine any further changes in the gut microbiota, when flies from the same population were transferred between two different diets in a short period of time. These flies formed the "ASG 2" treatment.

\section{$\mathrm{pH}$ and diet type on life history traits}

In order to test the effect of acidity on $D$. sechellia life history traits, we constructed three different diets of varying $\mathrm{pH}$. Flies were reared on one of three diets: ASG (for 11 of water: $85 \mathrm{~g}$ of sugar, $60 \mathrm{~g}$ of corn, $20 \mathrm{~g}$ of yeast, $10 \mathrm{~g}$ of agar and $25 \mathrm{ml}$ of nipagin), noni, or salak fruit. Morinda citrifolia is the diet of wild $D$. sechellia and has a low, acidic $\mathrm{pH}$ of 3.86 due to the high concentrations of both octanoic and hexanoic acids (Legal et al., 1994). The salak fruit diet was used as an alternative diet to the noni, as it also has a low, acidic $\mathrm{pH}$ at 3.59, but does not contain the toxic octanoic and hexanoic acids that are present in noni. These two diet types were compared to the typical laboratory diet of ASG, that has a higher, more alkaline $\mathrm{pH}$ than the fruit diets at 5.97.

\section{$\underline{\text { Risk of death before adulthood }}$}

The number of days was measured from day of female oviposition to day of adult emergence. Vials were checked at three time points within each day - 9am, 12pm and $5 \mathrm{pm}$ - and the cumulative number of adults emerged from each time point was scored $\left(N_{A S G}=76 ; N_{\text {Noni }}=60 ; N_{\text {Salak }}=125\right)$.

\section{Weight at different life stages}


In order to accurately determine the effect of acidity on life history, the weights of three different life stages were measured. For larval weight, vials were checked daily during the morning and any third instar larvae present were removed and washed with distilled water in order to remove any excess food. Larvae were grouped according to treatment and placed into the freezer at $-18^{\circ} \mathrm{C}$ for two hours. Later, larvae were removed and weighed using an Ohaus five place balance and their weight was recorded (in $\mathrm{mg}$ ) to four decimal places $\left(\mathrm{N}_{\mathrm{ASG}}=50 ; \mathrm{N}_{\text {Noni }}=50 ; \mathrm{N}_{\text {salak }}=50\right)$.

For the pupal and adult weights, vials were similarly checked daily at three time points - 9am, 12pm and 5pm - to check for any freshly pupated or newly emerged individuals. For the pupae, care was taken to remove pupae from the vials without damaging them $\left(N_{A S G}=50 ; N_{\text {Noni }}=50 ; N_{\text {salak }}=50\right)$. The adult flies were isolated as virgins and separated according to sex. Adults were placed into vials at a standard density of ten per vial and left for two hours to allow their wings to dry out and inflate. Two hours later, vials were placed into the freezer at $-18^{\circ} \mathrm{C}$ and left overnight. Pupae were grouped according to treatment and placed into the freezer at $-18^{\circ} \mathrm{C}$ for two hours. Later, the pupae and adults were removed and weighed using an Ohaus five place balance and their weight was recorded (in $\mathrm{mg}$ ) to four decimal places. In the adults, male and female measurements for each treatment were recorded and analysed separately (females: $\mathrm{N}_{\mathrm{ASG}}=53 ; \mathrm{N}_{\text {Noni }}=50 ; \mathrm{N}_{\text {salak }}=50 ;$ males: $\mathrm{N}_{\mathrm{ASG}}=45 ; \mathrm{N}_{\mathrm{Noni}}=45$; $N_{\text {salak }}=50$ ). This method of determining adult weight was used for both sections of the experiments.

\section{Experiment 2: Octanoic acid resistance in D. sechellia and D. melanogaster}

\section{$\underline{\text { Survival rate }}$}

To determine resistance to the toxic octanoic acid compound, the survival of the three different strains, outbred $D$. melanogaster, inbred $D$. melanogaster and $D$. sechellia, to exposure of differing concentrations of octanoic acid was measured. Newly emerged, virgin adults were isolated from the stock populations and transferred to fresh vials containing $25 \mathrm{ml}$ standard Drosophila media and left for 3 days to mature. During this time, $\geq 99 \%$ octanoic acid (purchased from Sigma-Aldrich) was diluted in distilled water to the following concentrations: $0 \%$ (distilled water), $1 \%, 5 \%, 10 \%, 25 \%$, $50 \%$. $30 \mu \mathrm{l}$ of the acid solute was pipetted onto the surface of a fresh vial containing 
$25 \mathrm{ml}$ standard Drosophila media. The vial was tipped to the side to ensure the acid covered the entire surface of the food media and left to dry for 2 hours. After this time, males and females were separated according to sex and placed at a standard density of 10 individuals per vial. The number of dead individuals was counted every 24 hours for a total period of 168 hours and the survival rate calculated $\left(\mathrm{N}_{\text {InbredDmel }}=50\right.$; NoutbredDmel $=50 ; N_{\text {Dsechellia }}=50$ for each acid concentration).

\section{Development time}

Mated adults at a density of five females and five males were placed onto fresh vials containing the following concentrations of octanoic acid: $0 \%$ (distilled water), $1 \%, 5 \%$, $10 \%, 25 \%, 50 \%$, to determine the effects of different concentrations of octanoic acid on development time. The pairs were left to oviposit. Development time was measured as the number of days from female oviposition to day of adult emergence. Vials were checked at three time points within each day - 9am, 12pm and 5pm - and the cumulative number of adults emerged from each time point was scored. Emergent adult flies from each time point were removed from the vial and placed into a fresh vial containing $25 \mathrm{ml}$ of standard Drosophila food (sample sizes are documented below.

\section{Experimental evolution of $D$. melanogaster lines exposed to $D$. sechellia gut microbiota}

To determine whether it is the gut microbiota that enables $D$. sechellia to become attracted to and feed on a diet that contains high levels of the toxic compound, octanoic acid, we created a series of experimental evolution lines. Using the stock population of outbred $D$. melanogaster, we added $D$. sechellia gut solute to the dietary media over several generations. Here, stock population $D$. sechellia that were continually reared on a noni diet, were first surface sterilised in $70 \%$ ethanol, rinsed in distilled water and air dried. The head was then removed. Two guts were dissected into each Eppendorf containing $250 \mu \mathrm{l}$ of sterile LB (Lysogeny Broth) broth. An equal number of males and females were used to ensure there were no sex-specific differences in the bacterial content. Gut tissue was homogenised with a sterile plastic pestle. $30 \mu \mathrm{l}$ of gut isolate was then pipetted onto the surface of a vial containing $25 \mathrm{ml}$ standard Drosophila media. The vial was tipped to the side to ensure the solute covered the entire surface of the food media and left to dry for 20 minutes. After this time, newly emerged, virgin males and females were isolated from the stock population of outbred $D$. melanogaster and placed at a standard density of 10 males and 10 females per vial. After pupae 
were seen in all vials, the adult flies were removed so the offspring and adults did not interbreed. Once the offspring had emerged as adults, a sub-sample of the first generation were isolated. Some of these individuals were harvested and their gut bacterial content and diversity analysed. The rest of the sub-sample of the first generation were placed into octanoic acid aversion trials (see protocol below). The remaining flies were placed onto a fresh vial similarly containing $25 \mathrm{ml}$ standard Drosophila media and $30 \mu \mathrm{l}$ of gut isolate. This process was repeated until the offspring reached the $10^{\text {th }}$ generation. Newly emerged adult offspring were harvested as before. The remaining adults were placed into octanoic acid aversion trials. Thus, the aversion trials were conducted on unselected stock individuals for each species, and the first and tenth generations of the experimental evolution lines.

\section{$\underline{\text { Octanoic acid aversion trials }}$}

In order to test whether experimental evolution of outbred $D$. melanogaster reared on a diet supplemented with $D$. sechellia gut microbiota reduces aversion to the toxic octanoic acid, aversion trials were performed, using a similar methodology to that utilised previously (e.g. Dekker et al., 2006). Newly emerged, virgin adults were isolated from the $D$. sechellia and outbred $D$. melanogaster stock populations, separated according to sex and placed into fresh vials containing $25 \mathrm{ml}$ standard Drosophila media. Similarly, newly emerged, virgin adults were isolated from the first generation (hereon known as Dmel 1) and $10^{\text {th }}$ generation (hereon known as Dmel 10) experimental evolution lines described above, separated according to sex and placed into fresh vials containing $25 \mathrm{ml}$ standard Drosophila media. Flies were left to mature for 3 days before being placed into an aversion arena (Figure 1). The aversion arena consisted of a standard petri dish (measuring $100 \mathrm{~mm} \times 15 \mathrm{~mm}$ ) containing two pieces $(10 \mathrm{~g})$ of standard Drosophila food located at opposite ends, with a marked line halfway across clearly showing the two separate sides. 10ul of concentrated octanoic acid was pipetted on to one of these pieces of food. An individual fly was gently aspirated into the centre of the arena and left to acclimatise for five minutes. After this time, the side on which the fly was located was scored as its preference, i.e., either the side with food containing octanoic acid, or without (female $\mathrm{N}_{\text {Dmel }}=50$, male $\mathrm{NDmel}=50$; female $\mathrm{N}_{\text {Dsech }}=50$, male $\mathrm{N}_{\text {Dsech }}=50 ;$ female $\mathrm{N}_{\text {Dmel1 }}=51$, male $\mathrm{N}_{\text {Dmel } 1}=50 ;$ female $\mathrm{N}_{\text {Dmel10 }}=$ 49, NDmel10 $=48$ ). 


\section{Bacterial analysis for all experiments}

Collected flies were first surface sterilised in $70 \%$ ethanol, rinsed in distilled water and air dried. The head was then removed. Two guts were dissected into each Eppendorf containing $250 \mu$ l of sterile LB (Lysogeny Broth) broth (Bertani, 2004). An equal number of males and females were used to ensure there were no sex-specific differences in the bacterial content. Gut tissue was homogenised with a sterile plastic pestle. $100 \mu \mathrm{l}$ of gut homogenate was pipetted onto BHI (Brain, Heart Infusion) agar (Atlas, 2004) and spread-plated using a sterile glass loop. BHI media was used as it was found to favour greater colony growth. Plates were left to air dry aseptically, before being closed and sealed with parafilm. Plates were incubated at $25^{\circ} \mathrm{C}$ for 72 hours, and bacterial load was quantified by performing CFU (Colony Forming Unit) counts.

Single colonies were isolated using a sterile $1 \mu \mathrm{l}$ loop and placed into an Eppendorf with $10 \mu$ l sterile water. PCR amplification was performed in a $25 \mu$ l reaction volume consisting of $10 \mu \mathrm{l}$ nuclease-free water, $13 \mu \mathrm{l}$ Taq green master mix, $0.5 \mu \mathrm{l}$ of forward primer 27F (5'- AGAGTTTGATCMTGGCTCAG-3') and reverse primer 1492R (5'GGTTACCTTGTTACGACTT-3') and $1 \mu$ l of template DNA. Thermal cycling was performed for 90 seconds at $95^{\circ} \mathrm{C}$ as initial denaturation, followed by 35 cycles of 30 $\mathrm{sec}$ at $95^{\circ} \mathrm{C}$ for denaturation, $30 \mathrm{sec}$ at $55^{\circ} \mathrm{C}$ as annealing, $90 \mathrm{sec}$ at $72{ }^{\circ} \mathrm{C}$ for extension, and final extension at $72{ }^{\circ} \mathrm{C}$ for $5 \mathrm{~min}$. $1500 \mathrm{bp} 16 \mathrm{~S}$ PCR products were purified with Ampure beads and subjected to Sanger sequencing. The resulting sequences were identified using NCBI BLAST against the nt database (Altschul et al., 1990).

\section{Statistical analysis}

All data were analysed using R (version 3.3.0; R Core Team, 2016). Larval, pupal and adult weight were analysed using separate General Linear Models (GLM). Adult weight was first analysed with both sexes grouped, before further analysis separated according to sex were performed. The aversion data was analysed using a binomial GLM. Variation in development time, survival response to octanoic acid and the risk of death before adulthood was analysed via Cox Proportional-Hazard Regressions. 
Development failure of flies was used as the 'event' for the risk of death before adulthood data. The Survdiff function was used to assess differences between two or more survival curves according to treatment. The coxph function was used to assess differences between treatments. This allowed treatments to be compared in a pairwise fashion, to ascertain whether all treatments differed, or whether any significant differences observed were derived from a single treatment.

\section{Results}

\section{Experiment 1: The changing gut microbiota of $D$. sechellia}

Bacterial colony growth was observed in all treatments, with both greater diversity and greater abundance of bacteria found in the ASG 1 and ASG 2 flies (Table 1). Flies analysed from these treatments were found to have Lactobacillus plantarum, Paenibacillus sp. and Bacillus cereus species present. In nearly all of the noni flies only L. plantarum was observed, with the exception of minor colony growth in two of the male replicates. Little difference was observed between the three different strains of $D$. sechellia, or between sexes. 
Table 1. Number of bacterial colonies isolated from the midgut of adult flies. Flies were first reared on ASG (represented by ASG 1), then moved onto noni, before being transferred back onto ASG (ASG 2).

\begin{tabular}{|c|c|c|c|c|c|c|}
\hline Diet & Strain & Replicate & Sex & $\begin{array}{c}L . \\
\text { plantarum }\end{array}$ & $\begin{array}{c}\text { Paenibacillus } \\
\text { sp. }\end{array}$ & $\begin{array}{l}\text { Bacillus } \\
\text { cereus }\end{array}$ \\
\hline ASG 1 & 0.21 & 1 & $F$ & $3.12 \times 10^{\wedge} 2$ & $0.20 \times 10^{\wedge} 1$ & 0 \\
\hline ASG 1 & 0.21 & 2 & $F$ & $1.82 \times 10^{\wedge} 2$ & $1.40 \times 10^{\wedge} 1$ & 0 \\
\hline ASG 1 & 0.21 & 1 & M & $1.58 \times 10^{\wedge} 2$ & 0 & $0.10 \times 10^{\wedge} 1$ \\
\hline ASG 1 & 0.21 & 2 & M & $2.50 \times 10^{\wedge} 1$ & $0.90 \times 10^{\wedge} 1$ & 0 \\
\hline ASG 1 & 0.07 & 1 & $F$ & $5.11 \times 10^{\wedge} 3$ & $1.81 \times 10^{\wedge} 2$ & $0.50 \times 10^{\wedge} 1$ \\
\hline ASG 1 & 0.07 & 2 & $F$ & $5.94 \times 10^{\wedge} 3$ & $1.23 \times 10^{\wedge} 2$ & $0.20 \times 10^{\wedge} 1$ \\
\hline ASG 1 & 0.07 & 1 & $\mathrm{M}$ & $4.88 \times 10^{\wedge} 3$ & $5.40 \times 10^{\wedge} 1$ & $0.70 \times 10^{\wedge} 1$ \\
\hline ASG 1 & 0.07 & 2 & M & $3.58 \times 10^{\wedge} 3$ & $1.75 \times 10^{\wedge}$ & $2.20 \times 10^{\wedge} 1$ \\
\hline ASG 1 & 0.08 & 1 & $\mathrm{~F}$ & $6.25 \times 10^{\wedge} 3$ & $2.02 \times 10^{\wedge} 2$ & $2.70 \times 10^{\wedge} 1$ \\
\hline ASG 1 & 0.08 & 2 & $F$ & $4.09 \times 10^{\wedge} 3$ & $1.96 \times 10^{\wedge} 2$ & $1.50 \times 10^{\wedge} 1$ \\
\hline ASG 1 & 0.08 & 1 & $M$ & $3.17 \times 10^{\wedge} 3$ & $2.70 \times 10^{\wedge} 1$ & $0.40 \times 10^{\wedge} 1$ \\
\hline ASG 1 & 0.08 & 2 & $\mathrm{M}$ & $2.89 \times 10^{\wedge} 3$ & $8.80 \times 10^{\wedge} 1$ & 0 \\
\hline Noni & 0.21 & 1 & $F$ & $2.72 \times 10^{\wedge} 3$ & 0 & 0 \\
\hline Noni & 0.21 & 2 & $F$ & $1.78 \times 10^{\wedge} 3$ & 0 & 0 \\
\hline Noni & 0.21 & 1 & $\mathrm{M}$ & $1.62 \times 10^{\wedge} 2$ & 0 & 0 \\
\hline Noni & 0.21 & 2 & M & $1.59 \times 10^{\wedge} 2$ & 0 & 0 \\
\hline Noni & 0.07 & 1 & $F$ & $1.43 \times 10^{\wedge} 3$ & 0 & 0 \\
\hline Noni & 0.07 & 2 & $F$ & $1.34 \times 10^{\wedge} 3$ & 0 & 0 \\
\hline Noni & 0.07 & 1 & M & $2.55 \times 10^{\wedge} 2$ & $1.50 \times 10^{\wedge} 1$ & 0 \\
\hline Noni & 0.07 & 2 & M & $1.92 \times 10^{\wedge} 2$ & $0.70 \times 10^{\wedge} 1$ & 0 \\
\hline Noni & 0.08 & 1 & $F$ & $4.5 \times 10^{\wedge} 2$ & 0 & 0 \\
\hline Noni & 0.08 & 2 & $F$ & $1.81 \times 10^{\wedge} 2$ & 0 & 0 \\
\hline Noni & 0.08 & 1 & M & $8.10 \times 10^{\wedge} 1$ & $0.50 \times 10^{\wedge} 1$ & 0 \\
\hline Noni & 0.08 & 2 & $M$ & $7.20 \times 10^{\wedge} 1$ & 0 & 0 \\
\hline ASG 2 & 0.21 & 1 & $F$ & $1.21 \times 10^{\wedge} 3$ & $2.40 \times 10^{\wedge} 1$ & 0 \\
\hline ASG 2 & 0.21 & 2 & $F$ & $1.45 \times 10^{\wedge} 3$ & $1.20 \times 10^{\wedge} 1$ & $0.10 \times 10^{\wedge} 1$ \\
\hline ASG 2 & 0.21 & 1 & M & $1.22 \times 10^{\wedge} 2$ & $0.20 \times 10^{\wedge} 1$ & $0.10 \times 10^{\wedge} 1$ \\
\hline ASG 2 & 0.21 & 2 & M & $2.31 \times 10^{\wedge} 2$ & $0.50 \times 10^{\wedge} 1$ & $0.20 \times 10^{\wedge} 1$ \\
\hline ASG 2 & 0.07 & 1 & $F$ & $4.51 \times 10^{\wedge} 2$ & $1.50 \times 10^{\wedge} 2$ & $0.70 \times 10^{\wedge} 1$ \\
\hline ASG 2 & 0.07 & 2 & $F$ & $5.22 \times 10^{\wedge} 2$ & $8.90 \times 10^{\wedge} 2$ & $0.20 \times 10^{\wedge} 1$ \\
\hline ASG 2 & 0.07 & 1 & $\mathrm{M}$ & $2.09 \times 10^{\wedge} 3$ & $2.90 \times 10^{\wedge} 1$ & $0.60 \times 10^{\wedge} 1$ \\
\hline ASG 2 & 0.07 & 2 & M & $2.87 \times 10^{\wedge} 3$ & $4.60 \times 10^{\wedge} 1$ & $1.30 \times 10^{\wedge} 1$ \\
\hline ASG 2 & 0.08 & 1 & $F$ & $3.22 \times 10^{\wedge} 3$ & $1.98 \times 10^{\wedge} 2$ & $2.90 \times 10^{\wedge} 1$ \\
\hline ASG 2 & 0.08 & 2 & $F$ & $2.46 \times 10^{\wedge} 3$ & $2.51 \times 10^{\wedge} 2$ & $2.20 \times 10^{\wedge} 1$ \\
\hline ASG 2 & 0.08 & 1 & M & $1.78 \times 10^{\wedge} 3$ & $1.12 \times 10^{\wedge} 2$ & $0.50 \times 10^{\wedge} 1$ \\
\hline ASG 2 & 0.08 & 2 & $M$ & $2.34 \times 10^{\wedge} 3$ & $1.43 \times 10^{\wedge} 2$ & $0.70 \times 10^{\wedge} 1$ \\
\hline
\end{tabular}




\section{$\underline{\text { Risk of death before adulthood }}$}

No significant difference was observed in the risk of death before adulthood between the ASG or noni treatments $\left(Z_{2}=0.373, P=0.709\right)$ (Figure 1). However, flies reared on salak had a significantly lower risk of death before adulthood than flies reared on noni $\left(Z_{2}=-2.187, P=0.028\right)$. A trend was observed in the risk of death before adulthood between ASG and salak treatments, with salak individuals exhibiting a higher risk of death before adulthood than ASG flies $\left(Z_{2}=-1.905 P=0.056\right)$.

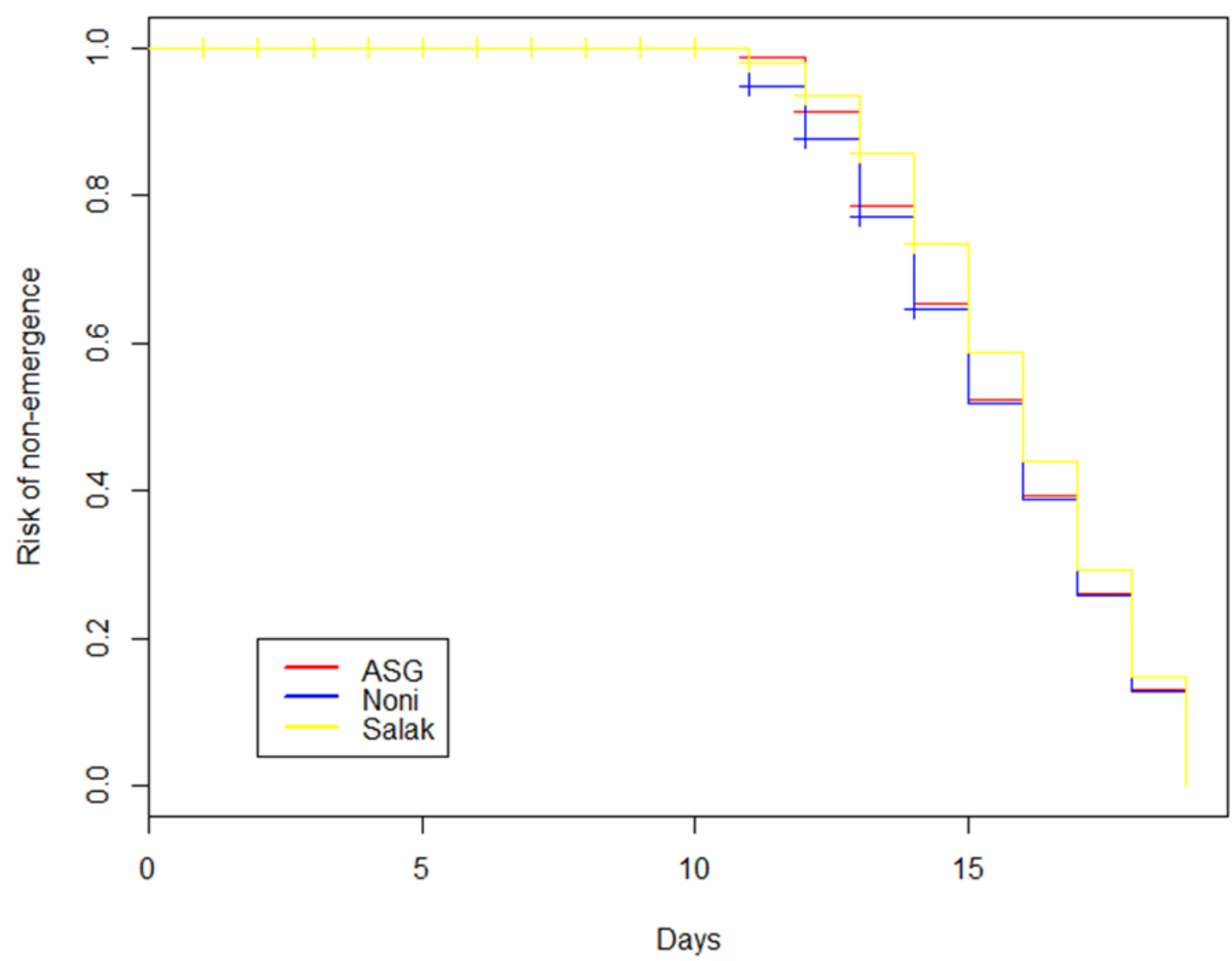

Figure 1. Development time failure, measured in days as the risk to die before adulthood, of $D$. sechellia. Eggs were reared under one of three different dietary treatments - either ASG, noni or salak diets. 


\section{$\underline{\text { Larval weight }}$}

No difference was observed in larval weight in any pairwise comparisons across all three treatments: $A S G$ and noni $\left(T_{2}=0.850, P=0.397\right)$, $A S G$ and salak $\left(T_{2}=0.335\right.$, $\mathrm{P}=0.738)$, noni and salak $\left(\mathrm{T}_{2}=-0.515, \mathrm{P}=0.608\right)$ (Figure 2$)$.

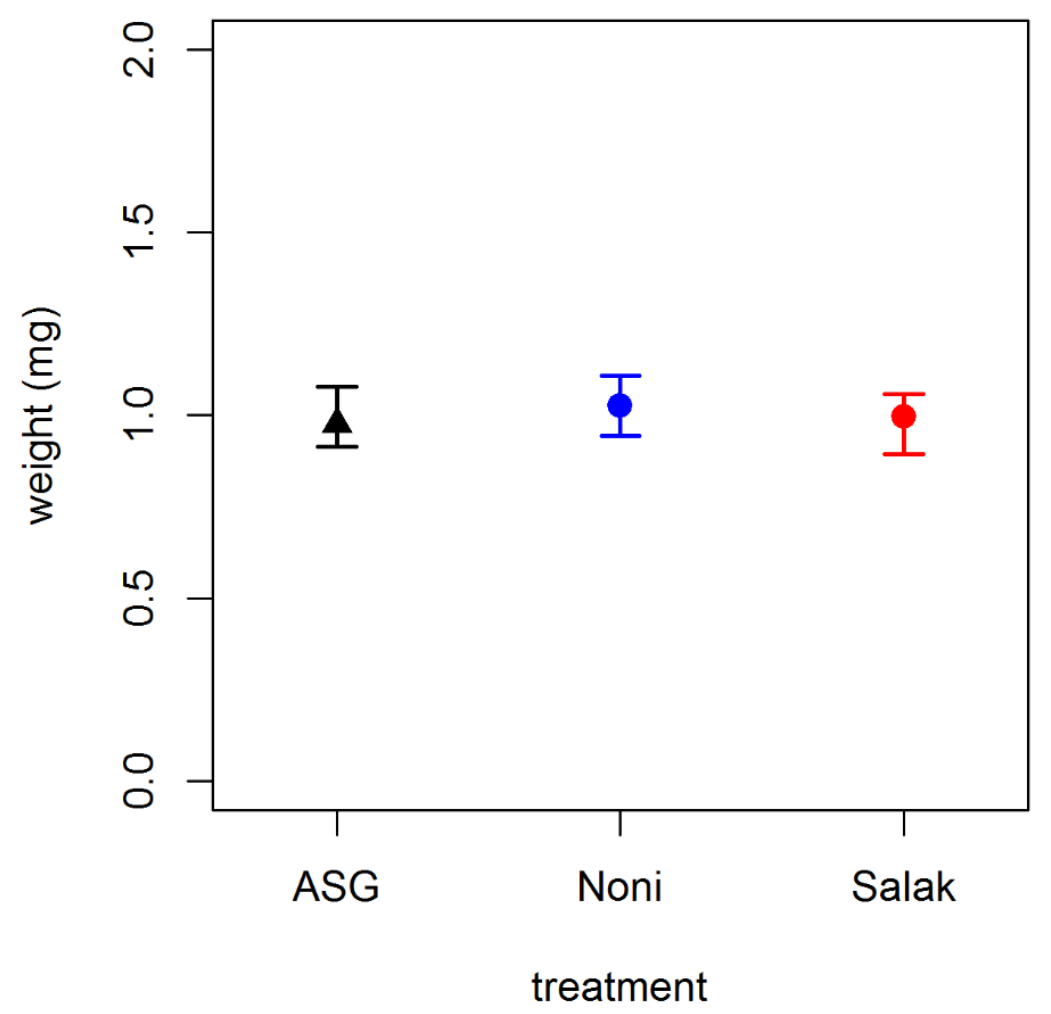

Figure 2. Weight $(\mathrm{mg})$ of third instar $D$. sechellia larvae reared on one of three different diet types - either ASG, noni or salak. No significant differences were found.

\section{$\underline{\text { Pupal weight }}$}

Pupae collected from the noni treatment weighed significantly less than pupae from the ASG treatment $\left(T_{2}=-8.961, P<0.001\right)$ (Figure 3). Similarly, pupae obtained from the salak treatment were found to weigh significantly less than the pupae from the ASG treatment $\left(T_{2}=-8.722, P<0.001\right)$. No difference in pupal weight was observed between pupae from the noni and salak treatments $\left(T_{2}=0.239, P=0.812\right)$. 


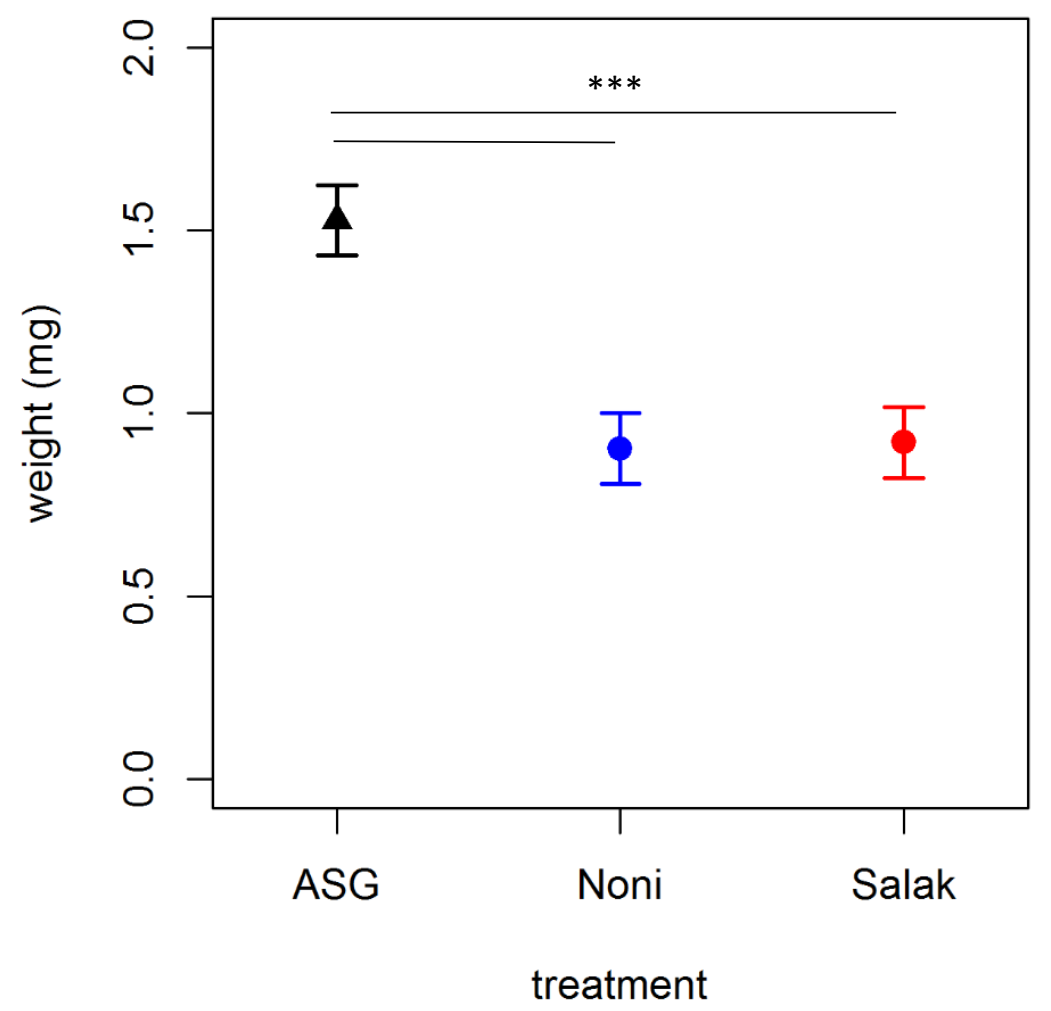

Figure 3. Weights $(\mathrm{mg})$ of $D$. sechellia pupae reared on one of three different dietary treatments - either ASG, noni or salak. Vials were checked at various time points for freshly pupated flies. Significant results are shown with *.

\section{$\underline{\text { Adult weight }}$}

Adult male flies were always found to weigh less than females $(P<0.001)$. When males and females are analysed separately, in male flies, significant differences were found across two comparisons, with both noni males and salak males weighing significantly more than ASG flies ( $\mathrm{T}_{2}=3.919, \mathrm{P}<0.001 ; \mathrm{T}_{2}=3.115, \mathrm{P}=0.002$, respectively) (Figure 4). However, no differences were found between the weights of noni and salak males $\left(\mathrm{T}_{2}=0.905, \mathrm{P}=0.366\right)$. In females, significant differences were found across all comparisons, with noni females weighing more than ASG females $\left(T_{2}=19.069\right.$, $P<0.001)$ and salak females $\left(T_{2}=-15.410, P<0.001\right)$. Similarly, salak females were also shown to weigh significantly more than ASG females $\left(T_{2}=3.435, P<0.001\right)$. 


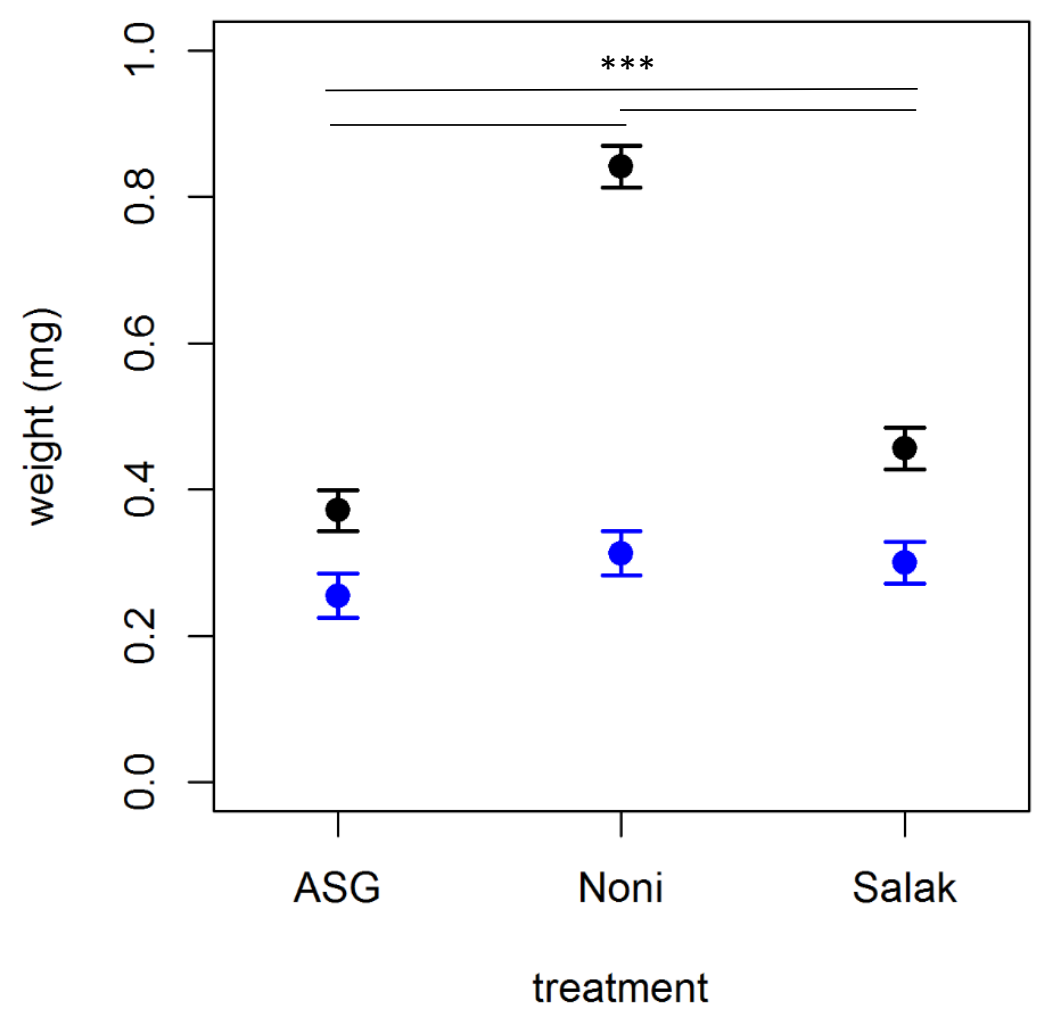

Figure 4. Weights $(\mathrm{mg})$ of both male and female, newly emerged adult $D$. sechellia flies reared on one of three different diet types - either ASG, noni or salak. Newly emerged adults were collected at various time points and allowed two hours for wing inflation. Male flies are shown here using the blue plots, whilst females are depicted in the black plots. Significant results are marked with a *.

\section{Bacterial analysis}

Bacterial colony growth was observed in all treatments, with a greater diversity of bacteria found in the flies that were reared on the ASG diet (Table 2). In all flies that were reared on the noni and salak treatments, only one kind of bacterial colony formed. Sanger sequencing data identified this as $L$. plantarum. In the ASG diet flies, $L$. plantarum was similarly observed, with Paenibacillus $s p$. and Bacillus cereus also found. Little difference was observed between the bacterial load of noni and salak reared flies, or between the different sexes. 
Table 2. Number of bacterial colonies isolated from the midgut of adult flies that were reared on one of three diets - ASG, noni or salak. Males and females were quantified separately.

\begin{tabular}{|c|c|c|c|c|c|}
\hline Diet & Replicate & Sex & L. plantarum & $\begin{array}{c}\text { Paenibacillus } \\
\text { sp. }\end{array}$ & B. cereus \\
\hline ASG & 1 & F & $2.52 \times 10^{\wedge} 2$ & $1.30 \times 10^{\wedge} 1$ & $0.20 \times 10^{\wedge} 1$ \\
& 2 & F & $2.91 \times 10^{\wedge} 2$ & $1.90 \times 10^{\wedge} 1$ & 0 \\
& 1 & M & $1.89 \times 10^{\wedge} 2$ & $2.30 \times 10^{\wedge} 1$ & 0 \\
& 2 & M & $2.34 \times 10^{\wedge} 2$ & $1.80 \times 10^{\wedge} 1$ & $0.60 \times 10^{\wedge} 1$ \\
\hline Noni & 1 & F & $2.85 \times 10^{\wedge} 2$ & 0 & 0 \\
& 2 & F & $3.19 \times 10^{\wedge} 2$ & 0 & 0 \\
& 1 & M & $1.51 \times 10^{\wedge} 2$ & 0 & 0 \\
& 2 & M & $1.99 \times 10^{\wedge} 2$ & 0 & 0 \\
\hline Salak & 1 & F & $1.31 \times 10^{\wedge} 2$ & 0 & 0 \\
& 2 & F & $1.66 \times 10^{\wedge} 2$ & 0 & 0 \\
& 1 & M & $2.12 \times 10^{\wedge} 2$ & 0 & 0 \\
& 2 & M & $2.45 \times 10^{\wedge} 2$ & 0 & 0 \\
\hline
\end{tabular}

\section{Experiment 2: Octanoic acid resistance in $D$. sechellia and D. melanogaster}

\section{Survival rate}

D. sechellia females reared on $0 \%$ octanoic acid exhibited significantly higher survival than females reared on the $1 \%$ concentration $\left(Z_{5}=3.340 ; P<0.001\right)$, the $5 \%$ concentration $\left(Z_{5}=3.936 ; P<0.001\right)$, the $25 \%$ treatment $\left(Z_{5}=4.740 ; P<0.001\right)$, or the $50 \%$ treatment $\left(Z_{5}=4.464 ; P<0.001\right)$ (Figure 5). However, no significant difference was observed between $0 \%$ and $10 \%$ treatments $\left(Z_{5}=1.664 ; P=0.096\right)$. Flies reared on the $10 \%$ treatment exhibited significantly higher survival than flies reared on the $25 \%$ treatment $\left(Z_{5}=3.564 ; P<0.001\right)$ or the $50 \%\left(Z_{5}=3.448 ; P<0.001\right)$.

In female inbred $D$. melanogaster, no difference was observed in the survival ability of $0 \%$ treated and $1 \%$ treated flies (Figure 5$)\left(Z_{5}=0.778 ; P=0.436\right)$, but $0 \%$ treated flies were significantly more able to survive than those reared on $5 \%\left(Z_{5}=4.020 ; P<0.001\right)$, $10 \%\left(Z_{5}=3.538 ; P<0.001\right), 25 \%\left(Z_{5}=9.943 ; P<0.001\right)$, or $50 \%\left(Z_{5}=13.075 ; P<0.001\right)$. Flies reared on the $1 \%$ treatment were significantly more able to survive than those at 
higher concentrations: 5\% $\left(Z_{5}=3.340 ; P<0.001\right), 10 \%\left(Z_{5}=2.836 ; P=0.004\right), 25 \%$ $\left(Z_{5}=9.689 ; \mathrm{P}<0.001\right)$, or $50 \%\left(Z_{5}=13.102 ; \mathrm{P}<0.001\right)$. Females reared at the $50 \%$ octanoic acid concentration exhibited significantly higher mortality than those reared at $25 \%\left(Z_{5}=5.919 ; P<0.001\right)$.

In female outbred D. melanogaster, flies reared at a $25 \%$ concentration had a significantly higher mortality rate than flies reared on any other treatment: $0 \%\left(Z_{5}=-\right.$ 7.205; $P<0.001), 1 \%\left(Z_{5}=-7.203 ; P<0.001\right), 5 \%\left(Z_{5}=-7.236 ; P<0.001\right), 10 \%\left(Z_{5}=-6.943\right.$; $P<0.001)$, including the $50 \%$ concentration $\left(Z_{5}=-2.789 ; P=0.005\right)$. Similarly, flies reared at $50 \%$ concentration had a significantly higher mortality rate than flies reared at $0 \%$ $\left(Z_{5}=-5.825 ; P<0.001\right), 1 \%\left(Z_{5}=-5.822 ; P<0.001\right), 5 \%\left(Z_{5}=-5.590 ; P<0.001\right)$ or $10 \%$ $\left(Z_{5}=-5.816 ; P<0.001\right)$.

When comparing the proportion of survived females across species, there is little difference in survival rates in $D$. sechellia across all octanoic acid concentrations, compared to inbred $D$. melanogaster (Figure 2). Here, females reared at higher concentrations exhibit a higher mortality, with all individuals recorded as dead in the $25 \%$ and $50 \%$ treatments, unlike in $D$. sechellia in which approximately half the sample were still alive at the end of the trial. The proportion of survived individuals is around 0.3 in outbred D. melanogaster at higher concentrations (25\% and 50\%), with individuals reared at low concentrations (0\%, 1\%,5\% and 10\%) exhibiting similar survival rates to $D$. sechellia. 


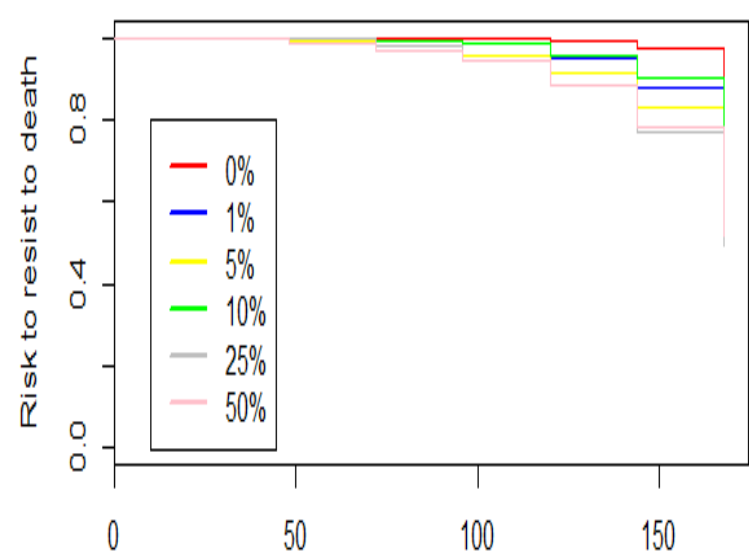

A
Hours

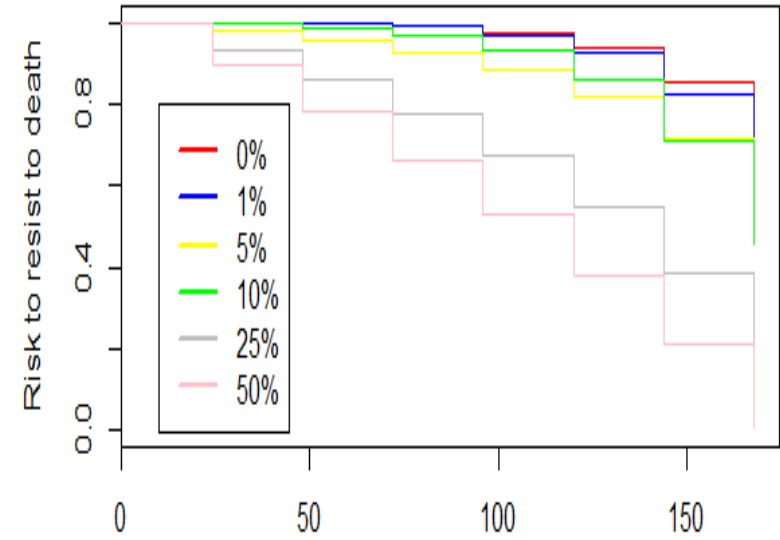

B

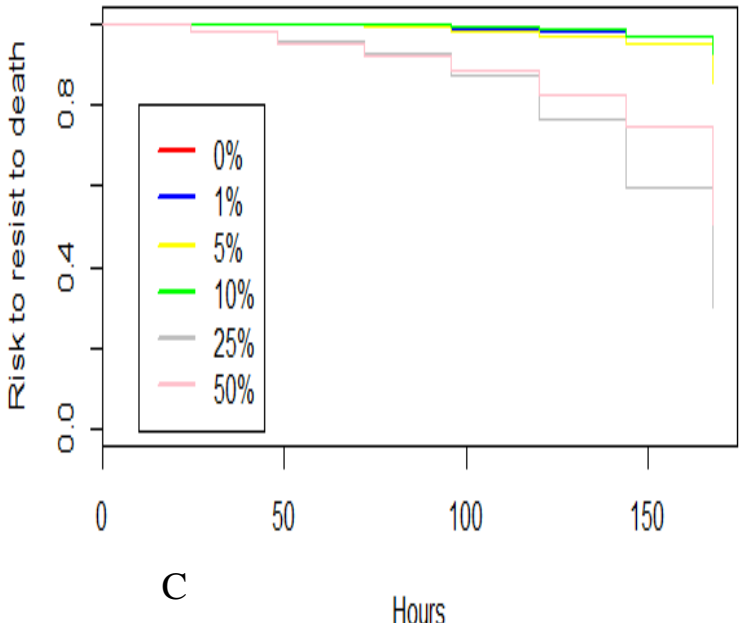

Hours

Hours

Figure 5. the proportion of survived female flies when exposed to standard Drosophila food containing differing concentrations of octanoic acid $0 \%, 1 \%, 5 \%, 10 \%, 25 \%, 50 \%$. The vials were checked every 24 hours with the last time point 168 hours, and the number of dead flies recorded. The graphs are arranged according to species: $D$. sechellia $(\mathrm{A})$, inbred $D$. melanogaster $(\mathrm{B})$ and outbred $D$. melanogaster (C). 
In contrast to female survival rates, in male $D$. sechellia, flies reared on the $0 \%$ acid treatment had equal survival rates to all other treatments, with no difference found compared to $1 \%$ reared flies $\left(Z_{5}=0.941 ; P=0.357\right), 5 \%\left(Z_{5}=1.341 ; P=0.180\right), 10 \%$ ( $\left.Z_{5}=-1.448 ; P=0.148\right), 25 \%\left(Z_{5}=0.808 ; P=0.419\right)$ or $50 \%\left(Z_{5}=-0.045 ; P=0.964\right)$ (Figure 6). Similar to the female $D$. sechellia, male flies reared on the $10 \%$ treatment were significantly more able to survive than those reared on $1 \%$ treatment $\left(Z_{5}=-2.239\right.$; $P=0.019)$, the $5 \%$ treatment $\left(Z_{5}=-2.691 ; P=0.007\right)$ and the $25 \%$ treatment $\left(Z_{5}=2.207\right.$; $\mathrm{P}=0.027)$.

In male inbred $D$. melanogaster flies, $0 \%$ reared flies had a significantly higher survival ability than $25 \%$ flies $\left(Z_{5}=7.830 ; P<0.001\right)$ and $50 \%$ flies $\left(Z_{5}=12.660 ; P<0.001\right)$. Flies reared on the $1 \%$ acid concentration had a higher proportion survived than all other concentrations, including 0\%: $\left(Z_{5}=3.013 ; P=0.002\right)$, 5\% $\left(Z_{5}=3.405 ; P<0.001\right), 10 \%$ $\left(Z_{5}=4.668 ; P<0.001\right), 25 \%\left(Z_{5}=9.556 ; P<0.001\right)$ and $50 \%\left(Z_{5}=13.244 ; P<0.001\right) . A$ significantly higher mortality was observed in the $25 \%$ and $50 \%$ treatments compared to both the $5 \%\left(Z_{5}=7.481 ; P<0.001, Z_{5}=12.430 ; P<0.001\right.$, respectively $)$ and the $10 \%$ treatment $\left(Z_{5}=6.311 ; P<0.001, Z_{5}=11.670 ; P<0.001\right.$, respectively).

In male outbred $D$. melanogaster, no significant difference in survival ability is found between flies reared at the lower concentrations. Flies reared at $10 \%$ concentration had a significantly higher mortality than those reared at $0 \%\left(Z_{5}=-3.120 ; P=0.001\right), 1 \%$ $\left(Z_{5}=-3.095 ; P=0.001\right)$ and $5 \%\left(Z_{5}=-2.618 ; P=0.008\right)$ but this was significantly lower than those reared at $25 \%$ and $50 \%\left(Z_{5}=5.770 ; P<0.001 ; Z_{5}=5.790 ; \quad P<0.001\right.$, respectively). Similarly, flies reared at $25 \%$ and $50 \%$ had a significantly higher mortality than those reared at $0 \%\left(Z_{5}=-7.061 ; P<0.001, Z_{5}=-7.074 ; P<0.001\right), 1 \%\left(Z_{5}=-7.035 ;\right.$ $\left.P<0.001, Z_{5}=-7.048 ; P<0.001\right)$ and 5\% $\left(Z_{5}=-7.026 ; P<0.001, Z_{5}=-7.040 ; P<0.001\right)$. All other pairwise comparisons for survival analysis are non-significant. 
When comparing the proportions of survived males across species, there is a vast difference in the final proportion of survived $D$. sechellia males compared to inbred $D$. melanogaster (Figure 6). Male inbred $D$. melanogaster are less able to survive than $D$. sechellia males, particularly when reared at high concentrations (e.g. $25 \%$ and $50 \%$ ). The survival ability of outbred $D$. melanogaster flies is better than that of inbred flies with a higher proportion survived at the end of the trial, even when males were placed at high concentrations, but the proportion was less than that of $D$. sechellia. 

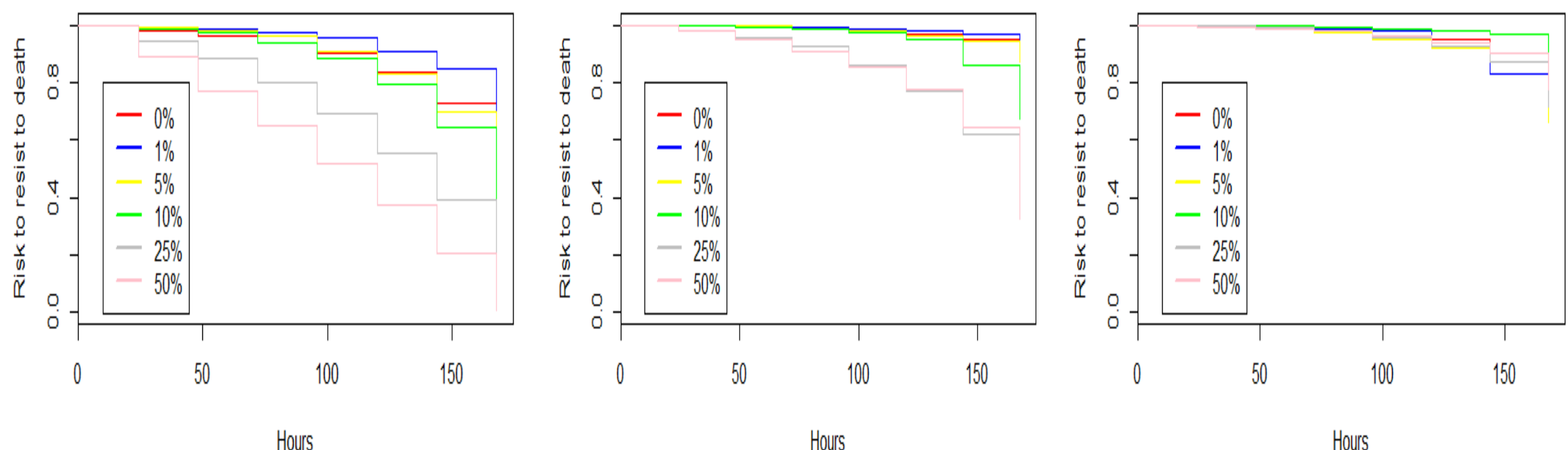

Figure 6. the proportion of survived male flies when exposed to standard Drosophila food containing differing concentrations of octanoic acid $0 \%, 1 \%, 5 \%, 10 \%, 25 \%, 50 \%$. The vials were checked every 24 hours with the last time point 168 hours, and the number of dead flies recorded. The graphs are arranged according to species: $D$. sechellia $(\mathrm{A})$, inbred $D$. melanogaster $(\mathrm{B})$ and outbred $D$. melanogaster $(\mathrm{C})$. 


\section{Development time}

In $D$. sechellia, no significant differences were found in the time taken to develop from egg to adulthood between any of the acid treatments (Figure 4). In particular, no significant difference was observed between the $0 \%$ and the $50 \%$ treatments $\left(\mathrm{F}_{5}=0.740 ; \mathrm{P}=0.459\right)$. However, in the inbred line of $D$. melanogaster, significant differences were observed between all treatments with flies reared on higher concentrations of acid taking longer to develop than those reared on smaller concentrations (Figure 7). Similarly, variation was seen in the time taken for outbred $D$. melanogaster individuals to develop when reared on different concentrations of acid (Figure 4). Flies reared on $0 \%$ acid concentration took significantly less time to develop than those reared on $1 \%$ treatment $\left(F_{5}=-7.930 ; P<0.001\right), 5 \%$ treatment $\left(F_{5}=-4.647\right.$; $\mathrm{P}<0.001)$, 10\% treatment $\left(\mathrm{F}_{5}=-8.679 ; \mathrm{P}<0.001\right), 25 \%\left(\mathrm{~F}_{5}=-7.808 ; \mathrm{P}<0.001\right)$ or $50 \%$ $\left(F_{5}=-4.900 ; \mathrm{P}<0.001\right)$. Similar to the inbred $D$. melanogaster, no significant differences were observed between $10 \%$ and $25 \%$ reared flies ( $\left.F_{5}=0.759 ; P=0.447\right), 10 \%$ and $50 \%$ flies ( $\left.F_{5}=1.733 ; P=0.083\right), 25 \%$ and $50 \%$ flies $\left(F_{5}=1.098 ; P=0.272\right)$. All other pairwise comparisons are non-significant. A full summary of all pairwise comparisons is provided in the appendix.

When comparing the development times across species, there is little variation in the time taken to develop on different concentrations of octanoic acid in $D$. sechellia (Figure 7). In comparison, inbred D. melanogaster take the shortest time to develop when reared at low concentrations, with time increasing as concentration increases. In outbred D. melanogaster, flies reared at low concentrations (e.g. $0 \%$ and $1 \%$ ) take the shortest time to develop, similar to inbred $D$. melanogaster. However, outbred $D$. melanogaster flies reared at the higher concentrations (e.g. $25 \%$ and $50 \%$ ) take less time than those reared at middling concentrations, such as $5 \%$ and $10 \%$. 


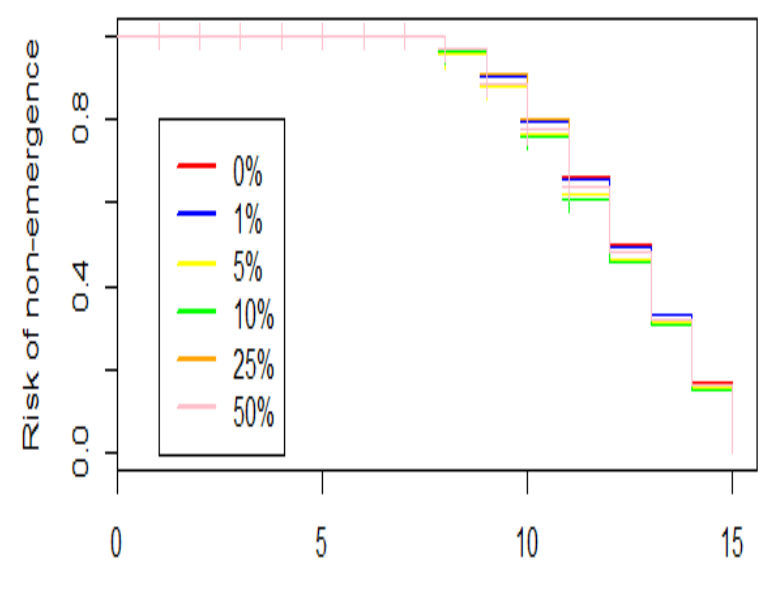

Days

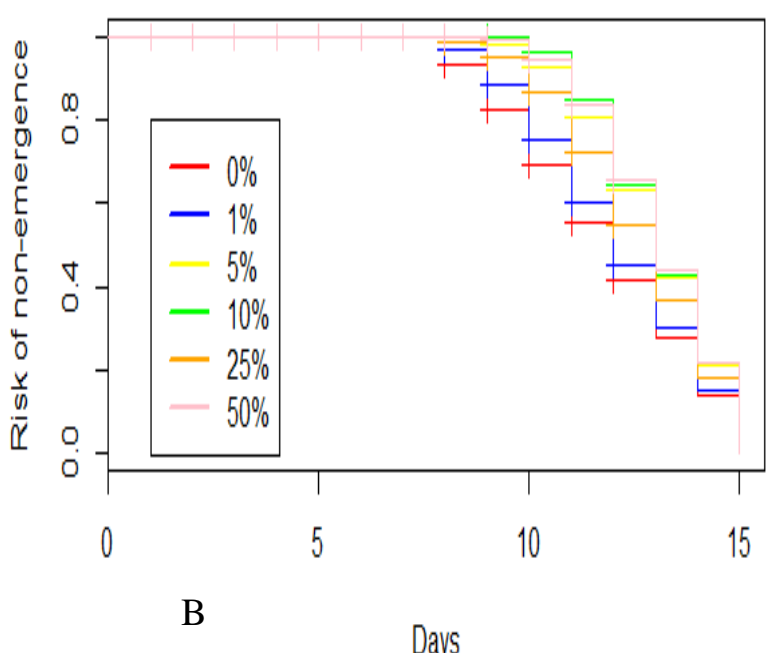

Days

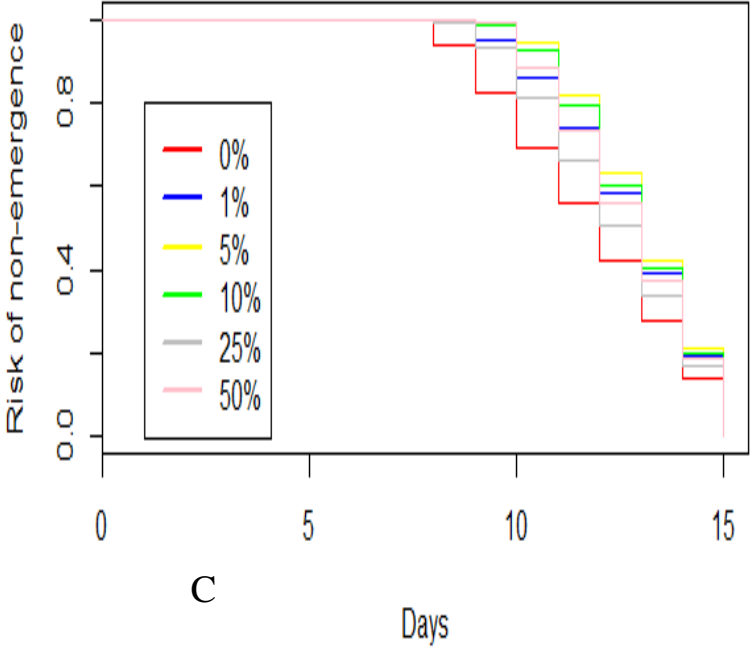

Days

A

Figure 7. Development time failure measured in days, as the risk to die before adulthood. Eggs were reared on standard Drosophila media that was supplemented with differing concentrations of octanoic acid $-0 \%, 1 \%, 5 \%, 10 \%, 25 \%, 50 \%$. The graphs are arranged according to species:

$D$. sechellia $(\mathrm{A})$, inbred $D$. melanogaster $(\mathrm{B})$ and outbred $D$. melanogaster $(\mathrm{C})$. 


\section{Female offspring adult weight}

In $D$. sechellia, female offspring reared on a $0 \%$ acid concentration weighed significantly more than females reared on 1\% ( $\left.T_{5}=-4.244 ; \mathrm{P}<0.001\right), 10 \%\left(\mathrm{~T}_{5}=-2.581\right.$; $\mathrm{P}=0.010)$ or $25 \%\left(\mathrm{~T}_{5}=-2.294 ; \mathrm{P}=0.022\right)$, but not $5 \%\left(\mathrm{~T}_{5}=-1.806 ; \mathrm{P}=0.071\right)$ or $50 \%$ $\left(T_{5}=1.109 ; P=0.2684\right)$ (Figure 8). Females reared on $50 \%$ acid concentration were significantly heavier than flies reared on all other concentrations, except $0 \%$.

In inbred $D$. melanogaster offspring, females reared on $0 \%$ acid concentration weighed significantly more than all other treatments, except 5\% $\left(T_{5}=-1.265 ; P=0.207\right): 1 \%\left(T_{5}=-\right.$ 7.877; $\mathrm{P}<0.001), 10 \%$ ( $\left.\mathrm{T}_{5}=-10.339 ; \mathrm{P}<0.001\right), 25 \%\left(\mathrm{~T}_{5}=-6.760 ; \mathrm{P}<0.001\right)$ and 50\% $\left(T_{5}=-19.368 ; P<0.001\right)$. Flies reared on $50 \%$ acid concentration weighed significantly less than all other treatments: $1 \%\left(T_{5}=9.573 ; P<0.001\right), 5 \%\left(T_{5}=14.628 ; P<0.001\right)$, $10 \%\left(T_{5}=7.163 ; P<0.001\right), 25 \%\left(T_{5}=10.603 ; P<0.001\right)$.

Female offspring of outbred D. melanogaster reared on $1 \%$ acid concentration weighed significantly more than all other concentrations: $0 \%\left(T_{5}=-4.853 ; P<0.001\right), 5 \%$ ( $\left.T_{5}=-9.595 ; \mathrm{P}<0.001\right), 10 \%\left(\mathrm{~T}_{5}=-14.142 ; \mathrm{P}<0.001\right), 25 \%\left(\mathrm{~T}_{5}=-13.217 ; \mathrm{P}<0.001\right)$ and $50 \%\left(T_{5}=-12.149 ; P<0.001\right)$. Females reared on $0 \%$ acid concentration weighed significantly more than $5 \%$ flies ( $\left.T_{5}=-4.704 ; P<0.001\right), 10 \%\left(T_{5}=-9.727 ; P<0.001\right), 25 \%$ $\left(T_{5}=-8.777 ; \mathrm{P}<0.001\right)$ and $50 \%\left(T_{5}=-7.661 ; \mathrm{P}<0.001\right)$.

Female weight has highly variable depending on species (Figure 8). Overall, female $D$. sechellia weighed less than inbred $D$. melanogaster flies, but there was less difference in weight across octanoic acid concentration. Female weight was more variable in both inbred and outbred $D$. melanogaster strains, with flies generally weighing more at low concentrations compared to high.

\section{Male offspring adult weight}

In male $D$. sechellia offspring, $0 \%$ flies weighed significantly less than $5 \%$ flies $\left(T_{5}=2.346 ; P=0.19\right), 10 \%$ flies $\left(T_{5}=3.401 ; P<0.001\right)$ and $25 \%$ flies $\left(T_{5}=2.630 ; P=0.008\right)$ (Figure 8). Male offspring reared at $1 \%$ concentration weighed significantly less than $5 \% \quad\left(T_{5}=2.797 ; P<0.005\right), 10 \% \quad\left(T_{5}=3.841 ; P<0.001\right)$ and $25 \%$ flies $\left(T_{5}=3.081 ;\right.$ $\mathrm{P}=0.002)$. 
In male inbred $D$. melanogaster offspring, unlike female flies, $0 \%$ flies weighed significantly more than all other treatments: $1 \%\left(\mathrm{~T}_{5}=-9.574 ; \mathrm{P}<0.001\right), 5 \%\left(\mathrm{~T}_{5}=-6.607\right.$; $\mathrm{P}<0.001), 10 \%\left(\mathrm{~T}_{5}=-16.772 ; \mathrm{P}<0.001\right), 25 \%\left(\mathrm{~T}_{5}=-7.971 ; \mathrm{P}<0.001\right)$ and $50 \%\left(\mathrm{~T}_{5}=-\right.$ 19.016; $P<0.001)$. Flies reared on $1 \%$ acid concentration weighed significantly more than $10 \%$ flies $\left(T_{5}=-5.603 ; P<0.001\right)$ and $50 \%$ flies $\left(T_{5}=-7.993 ; P<0.001\right)$ but weighed significantly less than flies reared on $5 \%$ acid concentration ( $\left.T_{5}=2.516 ; P=0.012\right)$.

In comparison, male outbred D. melanogaster offspring reared at $0 \%$ weighed significantly more than flies reared on $1 \%$ acid concentration $\left(\mathrm{T}_{5}=-7.564 ; \mathrm{P}<0.001\right)$, 5\% ( $\left.T_{5}=-5.830 ; P<0.001\right), 10 \%$ ( $\left.T_{5}=-13.483 ; P<0.001\right), 25 \%$ ( $\left.T_{5}=-11.467 ; P<0.001\right)$ and $50 \%\left(T_{5}=-12.639 ; P<0.001\right)$. Males reared on $1 \%$ and $5 \%$ acid concentrations weighed significantly more than flies reared on $10 \%\left(T_{5}=-6.028 ; P<0.001, T_{5}=-8.137\right.$; $\mathrm{P}<0.001$, respectively), 25\% ( $T_{5}=-3.826 ; \mathrm{P}<0.001, \mathrm{~T}_{5}=-5.929 ; \mathrm{P}<0.001$, respectively) and $50 \%$ ( $T_{5}=-4.840 ; P<0.001, T_{5}=-7.022 ; P<0.001$, respectively).

Similar to females, male weight was also highly variable across species (Figure 8). Male $D$. sechellia weight varied less than both inbred and outbred $D$. melanogaster males, with $D$. sechellia males always weighing more than both inbred and outbred $D$. melanogaster males at high concentrations, such as $50 \%$. 

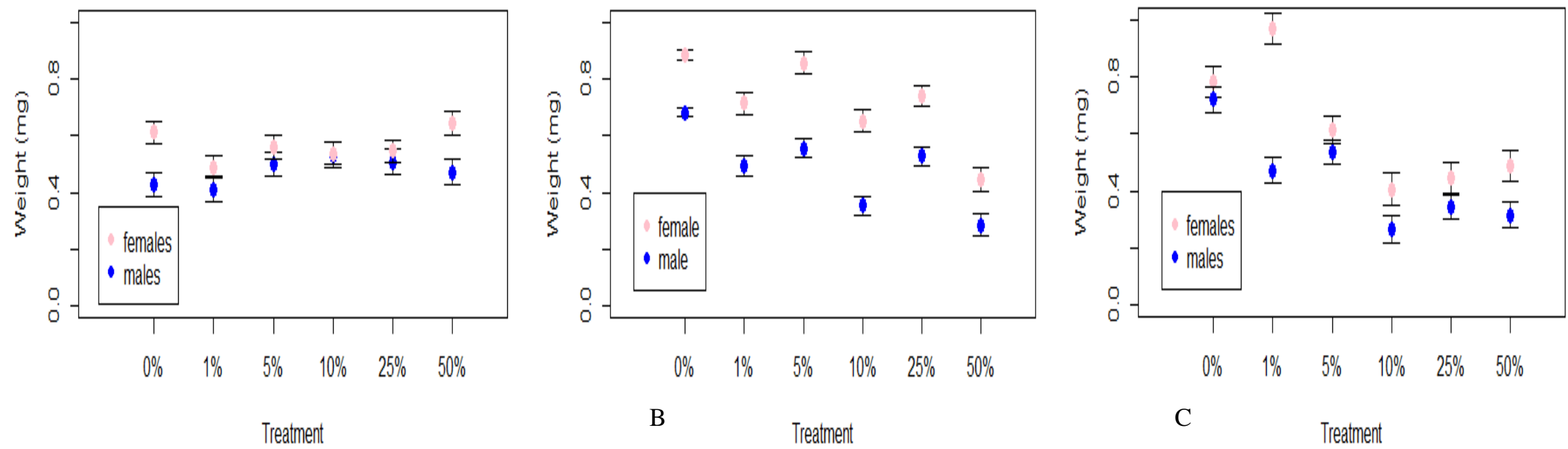

A

Figure 8. Weight of adult offspring (mg) of newly emerged adults, when their parents were reared on different concentrations of octanoic acid $0 \%, 1 \%, 5 \%, 10 \%, 25 \%, 50 \%$. The graphs are arranged according to species: $D$. sechellia $(\mathrm{A})$, inbred $D$. melanogaster (B) and outbred $D$. melanogaster $(\mathrm{C})$. Females are shown here by the pink dots and males represented by the blue. 


\section{Bacterial analysis}

Bacterial colony growth was observed in all treatments, with both greater diversity and greater abundance of bacteria found in the $D$. melanogaster inbred and outbred flies (Table 3). Sanger sequencing data identifies colony 1 as Lactobacillus plantarum; colony 2 as Paenibacillus sp. and colony 3 as Bacillus cereus. In all the D. sechellia adult flies and almost all the outbred $D$. melanogaster flies, only $L$. plantarum growth was observed. This is in comparison to the adult inbred $D$. melanogaster flies, which exhibited substantial growth of both L. plantarum, Paenibacillus $s p$. and B. cereus bacteria. Paenibacillus sp. and $B$. cereus colonies appear to be present in higher numbers when the $D$. melanogaster strains are reared at higher concentrations of the octanoic acid. The number of colonies identified of each bacterial species, from each Drosophila species, is included within the appendix (Table 4).

Table 3. Presence or absence of bacterial species detected in the midgut of adult $D$. sechellia and both outbred and inbred $D$. melanogaster flies, when reared on diets containing different concentrations of octanoic acid. Presence of a certain bacterial species is denoted with a tick $(\checkmark)$ and absence is with a cross $(\boldsymbol{x})$.

\begin{tabular}{|c|c|c|c|c|}
\hline Species & Concentration & L. plantarum & Paenibacillus sp. & B. cereus \\
\hline D. sechellia & $0 \%$ & $\checkmark$ & $x$ & $x$ \\
\hline D. sechellia & $1 \%$ & $\checkmark$ & $x$ & $x$ \\
\hline D. sechellia & $5 \%$ & $\checkmark$ & $x$ & $x$ \\
\hline D. sechellia & $10 \%$ & $\checkmark$ & $x$ & $x$ \\
\hline D. sechellia & $25 \%$ & $\checkmark$ & $x$ & $x$ \\
\hline D. sechellia & $50 \%$ & $\checkmark$ & $x$ & $x$ \\
\hline Outbred D. melanogaster & $0 \%$ & $\checkmark$ & $x$ & $x$ \\
\hline Outbred D. melanogaster & $1 \%$ & $\checkmark$ & $x$ & $x$ \\
\hline Outbred D. melanogaster & $5 \%$ & $\checkmark$ & $x$ & $x$ \\
\hline Outbred D. melanogaster & $10 \%$ & $\checkmark$ & $x$ & $x$ \\
\hline Outbred D. melanogaster & $25 \%$ & $\checkmark$ & $x$ & $x$ \\
\hline Outbred D. melanogaster & $50 \%$ & $\checkmark$ & $\checkmark$ & $x$ \\
\hline Inbred D. melanogaster & $0 \%$ & $\checkmark$ & $x$ & $x$ \\
\hline Inbred D. melanogaster & $1 \%$ & $\checkmark$ & $x$ & $x$ \\
\hline Inbred D. melanogaster & $5 \%$ & $\checkmark$ & $\checkmark$ & $\checkmark$ \\
\hline Inbred D. melanogaster & $10 \%$ & $\checkmark$ & $\checkmark$ & $\checkmark$ \\
\hline Inbred D. melanogaster & $25 \%$ & $\checkmark$ & $\checkmark$ & $\checkmark$ \\
\hline Inbred D. melanogaster & $50 \%$ & $\checkmark$ & $\checkmark$ & $\checkmark$ \\
\hline
\end{tabular}

$\underline{\text { Octanoic acid aversion trials }}$ 
Males and females were tested separately to determine if there was a difference in aversion rate according to sex. No difference was observed so subsequent analysis was performed with both sexes grouped together and separated according to species. Unselected $D$. sechellia (hereon known as Dsech ST) were found to prefer the food containing octanoic acid, in comparison to the unselected $D$. melanogaster stock population (hereon known as Dmel ST), which were significantly more averse $\left(\mathrm{F}_{5}=-\right.$ 2.124; $\mathrm{P}=0.027$ ). First generation D. melanogaster (Dmel 1) flies that had been reared on a diet supplemented with $D$. sechellia gut microbiota, were significantly more averse to the food containing octanoic acid than Dsech ST flies ( $\left.F_{5}=-2.541 ; P=0.011\right)$. There was no difference in aversion of octanoic acid found between Dsech ST and tenth generation $D$. melanogaster (Dmel 10) flies that had been reared on a diet supplemented with $D$. sechellia gut microbiota $\left(F_{5}=1.371 ; P=0.170\right)$. Dmel 10 flies were also found to be significantly less averse to octanoic acid than Dmel ST ( $F_{5}=2.889$; $\mathrm{P}=0.003)$; with no significant difference shown between Dmel ST and Dmel 1 flies $\left(\mathrm{F}_{5}=-\right.$ 0.973; $P=0.330$ ). Notably, Dmel 1 were found to be significantly more averse to the food containing octanoic acid than Dmel 10 flies $\left(F_{5}=3.774 ; \mathrm{P}<0.001\right)$.

Table 5. Proportions of times flies from each treatment group - D. sechellia, $D$. melanogaster, $D$. melanogaster $1^{\text {st }}$ generation and $D$. melanogaster $10^{\text {th }}$ generation - chose either the diet with octanoic acid present, or the diet without, when placed in the aversion assays.

\begin{tabular}{|c|c|c|}
\hline & \multicolumn{2}{|c|}{ Choice } \\
\hline Species & Proportion with octanoic acid & Proportion without \\
\hline D. sechellia & 0.60 & 0.40 \\
D. melanogaster & 0.35 & 0.65 \\
D. melanogaster 1st gen & 0.29 & 0.71 \\
D. melanogaster 10th gen & 0.69 & 0.31 \\
\hline
\end{tabular}

\section{Discussion}

Our results are the first to provide evidence that the gut microbiota may have played a role in host specialisation in Drosophila sechellia. We characterised the gut microbiota of wild-type, laboratory reared $D$. sechellia and demonstrated the impact that a changing diet has on gut microbiota. We found that by rearing $D$. sechellia on a fruit 
diet similar in nutritional properties to its natural host plant but without the toxins, we observe a microbiota of very similar diversity. We then showed the effect that altering the gut microbiota via diet has on subsequent life history traits, larval, pupal and adult weight, with little difference observed between larvae and pupae of all diet types. The only difference was that adults reared on the standard laboratory diet (ASG) weighed significantly less than noni reared flies. Noni flies had significantly higher development failure than salak flies, but they also weighed more at adult emergence, suggesting they have greater fitness.

As predicted, differences in survival ability, development time and resulting offspring adult weight were shown across the three different study groups $-D$. sechellia, inbred $D$. melanogaster and outbred $D$. melanogaster - when exposed to increasing concentrations of toxic, octanoic acid. $D$. sechellia generally exhibited higher survival rates in both males and females, when exposed to higher concentrations of octanoic acid. Inbred and outbred D. melanogaster exhibited similar survival abilities, with higher mortality seen at higher acid concentrations. The development time of offspring whose parents were reared on differing concentrations differed according to species, with $D$. sechellia and outbred $D$. melanogaster exhibiting a similar development time, and with more variance seen in inbred $D$. melanogaster. Offspring weight was also more variable in inbred and outbred $D$. melanogaster compared to $D$. sechellia, with both strains of $D$. melanogaster weighing more than $D$. sechellia at lower concentrations, but this effect was reversed at higher concentrations. Similar to previous results (Chandler et al., 2011), when reared on a standard Drosophila diet supplemented with octanoic acid, $D$. sechellia gut microbiota was found to be consistent to those isolated from the natural host plant, M. citrifolia. The bacteria isolated from $D$. sechellia was characterised as $L$. plantarum. This was also present in the gut of both inbred and outbred $D$. melanogaster, with $B$. cereus and Paenibacillus sp. also identified.

Little attention has been paid to the gut microbiota of $D$. sechellia, with the focus instead on genetic adaptation to its toxic host plant. Chandler et al. (2011) characterised the gut bacteria of wild $D$. sechellia found feeding on noni and determined that the natural gut microbiota of this species is dominated by a single Lactobacillales. This is in stark contrast to other fruit-feeding, closely related species of Drosophila that exhibit considerably greater bacterial diversity, such as wild $D$. 
melanogaster which host a number of bacterial genera, including Enterobacteriales, Burkholderiales and Pseudomonadales. Here, we show that the gut microbiota of wildtype $D$. sechellia is diverse when individuals are kept under laboratory conditions on a formulated diet. We determined that both males and female guts contain Paenibacillus sp. and Bacillus cereus. Although evidence has shown that the gut microbiota of laboratory reared species is considerably less diverse than their wild counterparts (Brummel et al., 2004; Roh et al., 2008), some studies do show deviation from the typically found bacterial genera of Lactobacillus, Acetobacters and Enterobacter in laboratory reared flies (e.g. Ren et al., 2007). It could therefore be argued that these genera of bacteria are present in wild populations of $D$. sechellia, but only thrive in great enough numbers for detection when placed onto a diet that encourages their growth. A bacterial pathogen, Paenibacillus species are known to be present in honeybee larvae and are responsible for colony collapse by causing American Foulbrood (e.g. Genersch, 2010).

Both Paenibacillus nanensis and $B$. cereus have been discovered in wild populations of Drosophila ananassae (Maji et al., 2013), although their function or effect on the host is as yet unknown. Presence of these pathogens in both strains on $D$. melanogaster may be due to the natural inability and avoidance of $D$. melanogaster to tolerate the octanoic acid. Although, previous studies have reported death on contact with either the noni or octanoic acid (R'Kha et al., 1991; Dekker et al., 2006), our study shows $D$. melanogaster are able to survive on moderate to high concentrations of the acid for a considerable period. This may be due to the strains of $D$. melanogaster used. Susceptibility to bacterial pathogens may be unwanted side effect of this survival ability, due to a weakened immune system. This may particularly be the case for the inbred strain of $D$. melanogaster, where a lack of genetic diversity may render individuals more susceptible to pathogen colonisation (e.g. Alarco et al., 2004).

When individuals are then transferred onto the natural host noni, the gut microbiota simplifies to a single species - Lactobacillus plantarum - as similarly shown in previous studies (Chandler et al., 2011). It could be suggested that colonies of Lactobacillus plantarum dominate when individuals are transferred onto noni, due to this bacterium acting as a detoxifying agent by metabolising the toxic compounds present in noni. In humans, L. plantarum is responsible for protecting the urogenital and intestinal tracts from infection from pathogenic bacteria (Reid and Burton, 2002). In Drosophila, L. 
plantarum has similarly been shown to protect against colonisation of pathogens in the gut (Ryu et al., 2008), by digesting sugars to produce lactic acid, which inhibits the growth of non-commensal organisms and promotes the growth of Lactobacilli that thrive in low pH conditions (e.g. Kleerebezem et al., 2003). It is also responsible for promoting larval growth when nutrients are scarce (Storelli et al., 2011), and plays a role in mating preferences (e.g. Sharon et al., 2010). Despite the clear role that $L$. plantarum plays on $D$. sechellia host physiology and likely role in digestion of toxic compounds, high levels of $L$. plantarum were also found when flies were reared on salak. Therefore, the dominance of $L$. plantarum may simply be due to the acidic conditions provided by both fruits. Further work is needed to elucidate the links between these two components.

The weight of individuals at different life stages greatly varied depending on the diet on which they were reared. At the larval stage, no difference in weights was observed across any of the treatments, yet at the pupal stage, ASG pupae weighed significantly more than those reared on noni or salak. As such high abundances of Lactobacillus plantarum were found in the gut across all treatments; it could be that $L$. plantarum, which is known to promote larval growth under conditions where nutrients are scarce, is compensating for the host developing on this laboratory formulated diet. In contrast, at adulthood, both male and female ASG reared flies weighed significantly less than both noni and salak flies. One reason for this difference in weights at adulthood may be due to the presence of $B$. cereus and Paenibacillus $s p$. in the adult ASG flies that are not present in individuals reared on noni or salak. B. cereus and Paenibacillus $s p$. have been reported in wild populations of $D$. ananassae (Maji et al., 2013), with some studies showing that the immune responses produced by $D$. melanogaster individuals in defence of the pathogen B. cereus, can have detrimental effects on life span (Ma et al., 2012; Ma et al., 2013). Therefore, it could be that the immune responses elicited by $D$. sechellia when individuals are reared on the less-preferred diet of ASG override the beneficial effects of $L$. plantarum to negatively affect adult weight.

As predicted, both female and male $D$. sechellia were able to survive on a diet containing the highest concentration of octanoic acid $(50 \%)$ with around $50 \%$ of individuals still alive at the end of the assay. Little differences were observed between any of the different acid concentrations, with the main difference that males reared on diets supplemented with $10 \%$ acid displayed a higher survival than those reared on a 
diet containing $0 \%$ octanoic acid. This suggests that addition of octanoic acid to $D$. sechellia is beneficial to survival, at least at lower concentrations. The ability of $D$. sechellia to survive at high concentrations of octanoic acid is somewhat to be expected as $M$. citrifolia's, main toxic constituent is octanoic acid, although there is some variation in the natural concentrations found, with some studies reporting $58 \%$ (Farine et al., 1996) and others 70\% (Pino et al., 2010).

Males and females from both the inbred and outbred lines of $D$. melanogaster were able to survive when placed onto a diet containing all concentrations of octanoic acid (Legal et al., 1994; Legal et al., 1999), however survival at high concentrations was lower than $D$. sechellia flies. This result is somewhat surprising as previous studies have noted that $D$. melanogaster dies upon contact with $M$. citrifolia, with most doing so within one hour (Legal et al., 1994; Legal et al., 1999). Survival of both sexes in the inbred $D$. melanogaster strain was significantly reduced at higher concentrations compared to a non-acidic diet, with nearly all individuals recorded dead at the end of the time period. The survival ability of outbred $D$. melanogaster was substantially better than the inbred strain, with only around $50 \%$ of females and $30 \%$ of males recorded as dead when reared at $50 \%$ acid concentration, at the end of the study. The outbred $D$. melanogaster strain may have a better ability to survive on the octanoic acid due to it being a wild-type strain and maintaining genetic diversity. Further, as $D$. sechellia is a sister species of $D$. melanogaster, the outbred or wild-type strain are more likely to share more genetic information than the inbred strain, including genes that underpin resistance to $M$. citrifolia. Animals with increased genetic diversity are also known to adapt to stress better than those with reduced genetic diversity (e.g. Bell, 2013).

The development time of offspring that emerged from adults that were reared on different concentrations of octanoic acid was measured. In $D$. sechellia, no differences were observed in the development time of offspring across any of the octanoic acid concentrations. Similarly, the outbred strain of $D$. melanogaster also exhibits little variation in development time between all octanoic acid concentrations, but flies reared at $0 \%$ took the shortest time to develop. This is in comparison to the inbred $D$. melanogaster, in which flies reared at the $0 \%$ acid concentration took significantly longer to develop than flies reared at higher concentrations. It is surprising that outbred $D$. melanogasterflies displayed a similar development time to $D$. sechellia. This is likely due to the conserved genetic diversity in the outbred line. Similar studies have shown 
that inbred $D$. melanogaster lines have a significantly reduced lifespan when exposed to dietary-restrictions, compared to that of outbred lines such as Dahomey (Grandison et al., 2009).

No difference was observed in adult weight of $D$. sechellia offspring, in parent flies reared on both $0 \%$ and $50 \%$ octanoic acid concentrations, with little variation observed in both males and females overall. In comparison, outbred $D$. melanogasterflies reared at higher concentrations, between $10 \%$ and $50 \%$, exhibited a significantly reduced weight in both sexes, with flies reared at $1 \%$ in females, and $0 \%$ in males weighing the most. Similar to the outbred line, male and female inbred $D$. melanogaster had a more variable weight range, with flies reared at $0 \%$ acid concentration weighing significantly more than flies reared at higher concentrations. As the concentration of octanoic acid in noni is between 58\% (Farine et al., 1996) and 70\% (Pino et al., 2010), the results for $D$. sechellia are contrary to expected. It could be predicted that $D$. sechellia reared at $0 \%$ concentration of octanoic acid would weigh less as this does not mimic its natural host plant. However, as this strain, although they are outbred, have been maintained in the laboratory since 1980, they may have become better adapted to the laboratory diet over time (e.g. Telonis-Scott et al., 2006). Variation between the weights of inbred and outbred strain flies is likely due to the conserved genetic diversity in the outbred line. Similar studies have shown that inbred $D$. melanogaster lines have a significantly reduced lifespan when exposed to dietary-restrictions, then that of outbred lines such as Dahomey (Grandison et al., 2009).

Drosophila melanogaster is known to die upon contact with the natural host plant of its sister species, $D$. sechellia, and as such has evolved mechanisms to detect and avoid this fruit (Legal et al., 1994; Legal et al., 1999). Using a series of aversion assays, we highlighted the differences in behavioural response to the presence of octanoic acid the main chemical component of noni and the chemical responsible for its pungent scent and toxic nature. Similar to previous results, stock population $D$. melanogaster were shown to be significantly more averse to octanoic acid than $D$. sechellia (Legal et al., 1999; Dekker et al., 2006). This is undoubtedly due to the presence of the OBPs (OBP57d and OBP57e) present in both sexes of D. sechellia that attract the flies to the octanoic acid within the fruit. Detection of the octanoic acid scent increases oviposition in females and so females seek the fruit in order to lay (Legal et al., 1999). 
A significant difference was found between $D$. sechellia and first-generation $D$. melanogaster flies that had been supplemented with $D$. sechellia gut microbiota. Here, first-generation $D$. melanogaster flies were significantly more averse to the food containing octanoic acid, and thus resembled the behaviour of standard stock population D. melanogaster (Legal et al., 1999; Dekker et al., 2006). However, no difference was found in preference for tenth-generation $D$. melanogaster flies compared with $D$. sechellia, showing that the aversion response was significantly reduced between first-generation and tenth-generation $D$. melanogaster individuals. This result potentially illustrates the first step in an organism specialising to a novel and toxic host. The genetic basis for aversion in $D$. melanogaster is due to the absence of the odorant-binding proteins (OBPs) OBP57d and OBP57e that are present on the gustatory sensilla on the legs of $D$. sechellia (e.g. Amlou et al., 1998; Jones, 2005; Matsuo et al., 2007; Dworkin and Jones, 2009). These enable the flies to detect the odour from up to 150 m away (R'Kha et al., 1991). However, no attention has been paid to the role of the gut microbiota in evolutionary preference and ability to synthesis toxic octanoic acid. We suggest that the gut microbiota can interact with the genetic mechanisms within the fly to override the natural aversion response, and thus contribute to the role of specialisation in this insect.

The gut microbiota of $D$. melanogaster is diverse but highly dependent on a number of factors, including diet (e.g. Sharon et al., 2010), age (Zerofsky et al., 2005), or strain (e.g. discussed in Heys et al., 2018b). When reared on a standard Drosophila diet (0\% acid concentration), our study determined that L. plantarum is present within both the $D$. melanogaster and $D$. sechellia gut. The results of the experimental evolution line of $D$. melanogaster supplemented with $D$. sechellia gut microbiota that we created, supports our argument that L. plantarum acts a detoxifying agent within the Drosophila gut. Octanoic acid is responsible for the majority of toxicity within the fruit (Farine et al., 1996; Jones, 1998). We predict the gut is able to withstand the toxicity of both the octanoic and hexanoic acids, via metabolising the toxic compounds into less harmful products which are able to be digested. Previous studies have shown that $\mathrm{pH}$ can determine the gut microbial composition (see Overend et al., 2016) and it could therefore be argued that sole presence of $L$. plantarum within the $D$. sechellia gut is due to $\mathrm{pH}$ alone. By determining the difference found between the first and tenth generation experimental evolution lines in aversion to octanoic acid, we can dispute 
the idea that the high colony numbers of $L$. plantarum is simply due to the increased $\mathrm{pH}$. For example, similar scenarios can be viewed in the mealworm, Tenebrio molitor, which detoxifies the cell walls of fungi and bacteria within its diet (Genta et al., 2006), and the coffee berry borer, Hypothenemus hampei, a specialist of coffee plants where Pseudomonas species within the gut microbiota metabolise caffeine - a toxic alkaloid (Ceja-Navarro et al., 2015).

This is the first time the gut microbiota of $D$. sechellia has been examined in laboratory conditions under different dietary treatments. Our results are in-keeping with others that characterise the microbiota of wild-caught $D$. sechellia (Chandler et al., 2011) despite the fact that our population has been reared in the laboratory on a formulated diet for a number of years. Here we demonstrate that when $D$. sechellia are reared on its natural host at any time point, a shift in the gut microbiota can be seen. Our results are the first to show the direct change in gut microbiota when the same individuals are moved between vastly different diets - the natural host plant and a laboratory diet. Although we determine these differences in microbiota across dietary treatments, further work is needed to disentangle the effect of $\mathrm{pH}$ on the gut microbiota, from a shift in microbiota that enables specialisation within this species. The present study provides evidence that the gut microbiota is responsible for specialisation in $D$. sechellia. $D$. melanogaster is known to be highly averse to the scent profile of octanoic acid, in comparison to $D$. sechellia in which it is a chemoattractant (Louis and David, 1986; R'Kha et al., 1991; Higa and Fuyama, 1993; Amlou et al., 1998; Legal et al., 1999). By creating experimental evolution lines of outbred $D$. melanogaster that are supplemented with $D$. sechellia gut microbiota, we have significantly reduced aversion of $D$. melanogaster to octanoic acid after only ten generations. In particular we suggest that Lactobacillus plantarum, the main bacterial constituent of the $D$. sechellia gut, acts as a detoxifying agent to metabolise the toxic octanoic acid compound - the main chemical present in the natural host plant, M. citrifolia. Little is known as to the origins of speciation of $D$. sechellia from its sister species, however, our results suggest that shifts in the gut microbiota may have led to ecological divergence, and later speciation, within this species. We have demonstrated an evolutionary shift in preference to food containing octanoic acid in $D$. melanogaster, to which it is naturally averse. Reducing aversion to a novel host plant could be the first step in ecological and evolutionary 
divergence. Further work is needed to understand how L. plantarum metabolises octanoic acid into presumably harmless components that can be utilised by the host.

\section{Acknowledgements}

This work was supported by the Natural Environment Research Council (grant number NE/L002450/1). The authors wish to thank Andrea Betancourt, Alistair Darby, Greg Hurst, and Tom Price, discussions with whom greatly improved this study.

\section{References}

Alarco, A.M., Marcil, A. Chen, J., Suter, B., Thomas, D. and Whiteway, M. (2004). Immune-deficient Drosophila melanogaster: a model for the innate immune response to human fungal pathogens. Journal of Immunology, 172, 5622-5628.

Amlou, M., Moreteau, B. and David, J.R. (1998). Genetic analysis of Drosophila sechellia specialization: oviposition behaviour toward the major aliphatic acids of its host plant. Behavioural Genetics, 28, 455-464.

Atlas, R.M. (2004). Handbook of Microbiological Media, (3 ed.). CRC Press. pp. 237247

Bell, G. (2013). Evolutionary rescue and the limits of adaptation. Philosophical Transactions of the Royal Society B Biological Sciences, 368, 20120080.

Bertani, G. (2004). Lysogeny at mid-twentieth century: P1, P2, and other experimental systems. Journal of Bacteriology, 186, 595-600.

Bolnick, D.I., Snowberg, L.K., Hirsch, P.E., Lauber, C.L., Knight, R., Caporaso, J.G. and Svanback, R. (2014). Individuals' diet diversity influences gut microbial diversity in two freshwater fish (threespine stickleback and Eurasian perch). Ecology Letters, 17, 979-987.

Brummel, T., Ching, A., Seroude, L., Simon, A.F. and Benzer, S. (2004). Drosophila lifespan enhancement by exogenous bacteria. Proceedings of the National Academy of Sciences of the United States of America, 101, 12974-12979.

Buchner, P. (1965). Endosymbioses of animals with plant microorganisms. Chichester, UK: John Wiley.

Ceja-Navarro, J.A., Vega, F.E., Karaoz, U., Hao, Z., Jenkins, S., Lim, H.C., Kosina, P., Infante, F. Northern, T.R. and Brodie, E.L. (2015). Gut microbiota mediate caffeine detoxification in the primary insect pest of coffee. Nature Communications, 6, 7618.

Chandler, J.A., Lang, J.M., Bhatnagar, S. Eisen, J.A. and Kopp, A. (2011). Bacterial communities of diverse Drosophila species: Ecological context of a host-microbe model system. PLOS Genetics, 7, e1002272. 
Cox, C.R. and Gilmore, M.S. (2007). Native microbial colonization of Drosophila melanogaster and its use as a model of Enterococcus faecalis pathogenesis. Infection and Immunity, 75, 1565-1576.

Dekker, T., Ibba, I., Siju, K.P., Stensmyr, M.C. and Hansson, B.S. (2006). Olfactory shifts parallel superspecialism for toxic fruit in Drosophila melanogaster sibling, $D$. sechellia. Current Biology, 16, 101-109.

Douglas, A.E. (1993). The nutritional quality of phloem sap utilized by natural aphid populations. Ecological Entomology, 18, 31-38.

Douglas, A.E., (1992). Microbial brokers of insect-plant interactions. Proceedings of eighth International Symposium on Insect-Plant Relationships, Kluwer, Dordrecht, pp. 329-336.

Dworkin, I. and Jones, C.D. (2009). Genetic changes accompanying the evolution of host specialization in Drosophila sechellia. Genetics, 181, 721-736.

Edger, P.P., Heidel-Fischer, H.M., Bekaert, M., Rota, J., Glöckner, G. Platts, A.E., Heckel, D.G., Der, J.P., Wafula, E.K., Tang, M., Hofberger, J.A., Smithson, A., Hall, J.C., Blanchette, M., Bureau, T.E., Wright, S.I., dePamphilis, C.W., Schranz, M.E., Barker, M.S., Conant, G.C., Wahlberg, N., vogel, H., Pires, J.C. and Wheat, C.W. (2015). The butterfly plant arms-race escalated by gene and genome duplications. PNAS, 27, 8362-8366.

Farine, J.P., Legal, L., Moreteau, B., and Le Quere, J.L. (1996). Volatile components of ripe fruit of Morinda citrifolia and their effects on Drosophila. Phytochemistry, 41, 433-438.

Genersch, E. (2010). American Foulbrood in honeybees and its causative agent, Paenibacillus larvae. Journal of Invertebrate Pathology, 103, S10-S19.

Genta, F.A., Dillon, R.J., Terra, W.R. and Ferreira, C. (2006). Potential role for gut microbiota in cell wall digestion and glucoside detoxification in Tenebrio molitor larvae. Journal of Insect Physiology, 52, 593-601.

Grandison, R.C., Wong, R., Bass, T.M., Partridge, L. and Piper, M.D.W. (2009). Effect of a standardised dietary restriction protocol on multiple laboratory strains of Drosophila melanogaster. PLOS ONE, 4, 1, e4067.

Gündüz, E.A. and Douglas, A.E. (2009). Symbiotic bacteria enable insect to use a nutritionally inadequate diet. Proceedings of the Royal Society B, 276, 987-991.

Harada, E., Haba, D., Aigaki, T. and Matsuo, T. (2008). Behavioural analyses of mutants for two odorant-binding protein genes, Obp57d and Obp57e, in Drosophila melanogaster. Genes and Genetic Systems, 83, 257-264.

Harada, E., Nakagawa, J., Asano, T., Taoka, M., Sorimachi, H., Ito, Y., Aigaki, T. and Matsuo, T. (2012). Functional evolution of duplicated odorant-binding protein genes, Obp57d and Obp57e, in Drosophila. PLoS ONE 7, e29710.

Herrera, C.M. and Pellmyr, O. (2009). Plant animal interactions: an evolutionary approach. Blackwell Science Ltd. 
Heys, C., Lizé, A., Colinet, H., Price, T.A.R.P., Prescott, M., Ingleby, F. and Lewis, Z. (2018a). Evidence that the microbiota counteracts male outbreeding strategy by inhibiting sexual signalling in females. Frontiers in Ecology and Evolution, 6, 29.

Heys, C., Lizé, A., Blow, F., White, L., Darby, A. and Lewis, Z. (2018b). The effect of gut microbiota elimination in Drosophila melanogaster: A how-to guide for hostmicrobiota studies. Ecology and Evolution, 8, 4150-4161.

Higa, I. and Fuyama, Y. (1993). Genetics of food preference in Drosophila sechellia. I. Responses to food attractants, Genetica, 88, 129-136.

Jones, C.D. (1998). The genetic basis of Drosophila sechellia's resistance to a host plant toxin. Genetics, 149, 1899-1908.

Jones, C.D. (2005). The genetics of adaptation in Drosophila sechellia. Genetics of Adaptation, 3, 137-145.

Kleerebezem, M., Boekhorst, J., van Kranenburg, R., Molenaar, D., Kuipers, O.P., Leer, R., Tarchini, R., Peters, S.A., Sandbrink, H.M., Fiers, M.W.E.J., Stiekema, W., Lankhorst, R.M.K., Bron, P.A., Hoffer, S.M., Groot, M.N.N., Kerkhoven, R., de Vries M., Ursing, B., de Vos, W.M., Siezen, R.J. (2003). Complete genome sequence of Lactobacillus plantarum WCFS1. Proceedings of the Natural Academy of Sciences USA, 100, 1990-1995

Labeur, C., Dallerac, R., and Wicker-Thomas, C. (2002). Involvement of Desat1 gene in the control of Drosophila melanogaster pheromone biosynthesis. Genetica, 114, 269-274.

Lachaise, D., Cariou, M.L., David, J.R., Lemeunier, F., Tsacas, L., and Ashburner, M. (1988). Historical biogeography of the Drosophila melanogaster species subgroup. Evolutionary Biology, 22, 159-225.

Legal, L., Chappe, B. and Jallon, J.M. (1994) Molecular basis of Morinda citrifolia, (L.): toxicity on Drosophila. Journal of Chemical Ecology, 20, 1931-1943.

Legal, L., David, J.R. and Jallon, J.M. (1992). Toxicity and attraction effects produced by Morinda citrifoliafruits on the Drosophila melanogastercomplex of species. Chemoecology, 3, 125-129.

Legal, L., Moulin, B. and Jallon, J.M. (1999) The relation between structures and toxicity of oxygenated aliphatic compounds homologous to the insecticide octanoic acid and the chemotaxis of two species of Drosophila. Pesticide Biochemical Physiology, 65, 90-101.

Lizé, A., McKay, R. and Lewis, Z. (2014). Kin recognition in Drosophila: the importance of ecology and gut microbiota. ISME, 8, 469-477.

Louis, J. and David, J.R. (1986). Ecological specialization in the Drosophila melanogaster species subgroup: a case study of $D$. sechellia. Acta Oceologica, 7, 215229.

Ma, J. Benson, A.K., Kachman, S.D., Hu, Z. and Harshman, L.G. (2012). Drosophila melanogaster selection for survival of Bacillus cereus infection: life history trait indirect responses. International Journal of Evolutionary Biology, 935970 
Ma, J. Benson, A.K., Kachman, S.D., Jacobsen, D.J. and Harshman, L.G. (2013). Drosophila melanogaster selection for survival after infection with Bacillus cereus spores: evolutionary genetic and phenotypic investigations of respiration and movement. International Journal of Evolutionary Biology, 576452.

Maji, P., Chakrabarti, C. and Chatterjee, S. (2013). Isolation and characterisation of symbiotic bacteria from the midgut of natural population of Drosophila ananassae. International Journal of Pharma and Bio Sciences, 4, 697-707.

Matsuo, T.S. Sugaya, S., Yasukawa, J., Aigaki, T. and Fuyama, Y. (2007). Odorantbinding proteins OBP57d and OBP57e affect taste perception and host-plant preference in Drosophila sechellia. PLoS Biology, 5, e118.

Morrow, J.L., Frommer, M., Shearman, D.C.A. and Riegler, M. (2015). The microbiome of field-caught and laboratory-adapted Australian Tephritid fruit fly species with different host plant use and specialisation. Microbial Ecology, 70, 498-508.

Overend, G., Luo, Y., Henderson, L., Douglas, A.E., Davies, S.A. and Dow, J.A.T. (2016). Molecular mechanism and functional significance of acid generation in the Drosophila midgut. Scientific Reports, 6, 27242.

Pino, J.A., Marques, E., Quijano, C.E. and Castro, D. (2010). Volatile compounds in noni (Morinda citrifolia L.) at two ripening stages. Ciência e Tecnologia de Alimento, 30, 183-187.

R Core Team (2016). R: A language and environment for statistical computing. $R$ Foundation for Statistical Computing, Vienna, Austria

R'Kha, S., Capy, P. and David, J.R. (1991). Host-plant specialization in the Drosophila melanogaster species complex: a physiological, behavioural, and genetical analysis. Proceedings of the Natural Academy of Sciences USA, 88, 1835-1839.

Reid, G. and Burton, J. (2002). Use of Lactobacillus to prevent infection by pathogenic bacteria. Microbes and Infection, 4, 319-324.

Ren, C., Webster, P., Finkel, S.E. and Tower, J. (2007). Increased internal and external bacterial load during Drosophila aging without life-span trade-off. Cell Metabolism, 6,144-152.

Roh, S.W., Nam, Y.D., Chang, H.W., Kim, M.S., Ryu, J.H., Kim, S.H., Lee, W.J. and Bae, J.W. (2008). Phylogenetic characterization of two novel commensal bacteria involved with innate immune homeostasis in Drosophila melanogaster. Applied Environmental Microbiology, 74, 6171-6177.

Rohlfs, M. and Kürschner, L. (2010). Saprophagous insect larvae, Drosophila melanogaster, profit from increased species richness in beneficial microbes. Journal of Applied Entomology, 134, 667-671.

Ryu, J.H., Kim, S.H., Lee, H.Y., Bai, J.Y., Nam, Y.D., Bae, J.W., Lee, D.G., Shin, S.C., Ha, E.M. and Lee W.J. (2008). Innate immune homeostasis by the homeobox gene Caudal and commensal-gut mutualism in Drosophila. Science, 319, 777-782.

Sandström, J. and Moran, N.A. (1999). How nutritionally imbalanced is phloem sap for aphids? Entomologia Experimentalis et Applicata, 91, 203-210. 
Sandström, J. (2000). Nutritional quality of phloem sap in relation to host plantalternation in the bird cherry-oat aphid. Chemoecology, 10, 17-24.

Telonis-Scott, M., Guthridge, K.M. and Hoffmann, A.A. (2006). A new set of laboratoryselected Drosophila melanogaster lines for the analysis of desiccation resistance: response to selection, physiology and correlated responses. Journal of Experimental Biology, 209, 1837-1847.

Vilanova, C., Baixeras, J., Latorre, A. and Porcar, M. (2016). The generalist inside the specialist: gut bacterial communities of two insect species feeding on toxic plants are dominated by Enterococcus sp. Frontiers in Microbiology, 7, 1005.

Zerofsky, M., Harel, E., Silverman, N. and Tatar, M. (2005). Aging of the innate immune response in Drosophila melanogaster. Ageing Cell, 4, 103-108.

\section{Appendix}

Table 1. Sample sizes for the measurements of development time for each species and strain measured: $D$. sechellia, $D$. melanogaster inbred, $D$. melanogaster outbred.

\begin{tabular}{|c|c|c|}
\hline Species & Treatment & Sample size \\
\hline Inbred D. melanogaster & $0 \%$ & 279 \\
Inbred D. melanogaster & $1 \%$ & 275 \\
Inbred D. melanogaster & $5 \%$ & 202 \\
Inbred D. melanogaster & $10 \%$ & 157 \\
Inbred D. melanogaster & $25 \%$ & 117 \\
Inbred D. melanogaster & $50 \%$ & 111 \\
Outbred D. melanogaster & $0 \%$ & 235 \\
Outbred D. melanogaster & $1 \%$ & 213 \\
Outbred D. melanogaster & $5 \%$ & 185 \\
Outbred D. melanogaster & $10 \%$ & 124 \\
Outbred D. melanogaster & $25 \%$ & 121 \\
Outbred D. melanogaster & $50 \%$ & 60 \\
D. sechellia & $0 \%$ & 152 \\
D. sechellia & $1 \%$ & 139 \\
D. sechellia & $5 \%$ & 198 \\
D. sechellia & $10 \%$ & 136 \\
D. sechellia & $25 \%$ & 174 \\
D. sechellia & $50 \%$ & 176 \\
\hline
\end{tabular}


bioRxiv preprint doi: https://doi.org/10.1101/526517; this version posted January 22, 2019. The copyright holder for this preprint (which was not certified by peer review) is the author/funder, who has granted bioRxiv a license to display the preprint in perpetuity. It is made available under aCC-BY-NC-ND 4.0 International license. 
Table 2. Sample sizes for the measurements of adult emergence weight for each species, sex and strain measured: D. sechellia, D. melanogaster inbred, $D$. melanogaster outbred.

\begin{tabular}{|c|c|c|c|}
\hline Species & Sex & Treatment & Sample size \\
\hline Inbred D. melanogaster & Female & $0 \%$ & 200 \\
Inbred D. melanogaster & Female & $1 \%$ & 275 \\
Inbred D. melanogaster & Female & $5 \%$ & 46 \\
Inbred D. melanogaster & Female & $10 \%$ & 45 \\
Inbred D. melanogaster & Female & $25 \%$ & 50 \\
Inbred D. melanogaster & Female & $50 \%$ & 44 \\
Inbred D. melanogaster & Male & $0 \%$ & 235 \\
Inbred D. melanogaster & Male & $1 \%$ & 50 \\
Inbred D. melanogaster & Male & $5 \%$ & 50 \\
Inbred D. melanogaster & Male & $10 \%$ & 50 \\
Inbred D. melanogaster & Male & $25 \%$ & 50 \\
Inbred D. melanogaster & Male & $50 \%$ & 43 \\
Outbred D. melanogaster & Female & $0 \%$ & 59 \\
Outbred D. melanogaster & Female & $1 \%$ & 52 \\
Outbred D. melanogaster & Female & $5 \%$ & 69 \\
Outbred D. melanogaster & Female & $10 \%$ & 50 \\
Outbred D. melanogaster & Female & $25 \%$ & 50 \\
Outbred D. melanogaster & Female & $50 \%$ & 50 \\
Outbred D. melanogaster & Male & $0 \%$ & 51 \\
Outbred D. melanogaster & Male & $1 \%$ & 49 \\
Outbred D. melanogaster & Male & $5 \%$ & 56 \\
Outbred D. melanogaster & Male & $10 \%$ & 45 \\
Outbred D. melanogaster & Male & $25 \%$ & 50 \\
Outbred D. melanogaster & Male & $50 \%$ & 53 \\
D. sechellia & Female & $0 \%$ & 52 \\
D. sechellia & Female & $1 \%$ & 51 \\
D. sechellia & Female & $5 \%$ & 50 \\
D. sechellia & Female & $10 \%$ & 51 \\
D. sechellia & Female & $25 \%$ & 51 \\
D. sechellia & Female & $50 \%$ & 52 \\
D. sechellia & Male & $0 \%$ & 54 \\
D. sechellia & Male & $1 \%$ & 51 \\
D. sechellia & Male & $5 \%$ & 51 \\
D. sechellia & Male & $10 \%$ & 53 \\
D.lia & Male & $25 \%$ & 53 \\
Male & $50 \%$ & 50 \\
\hline
\end{tabular}

